\title{
A Relation-algebraic Approach to Simple Games
}

\author{
Rudolf Berghammer ${ }^{\mathrm{a}}$, Stefan Bolus ${ }^{\mathrm{a}}$, Agnieszka Rusinowska ${ }^{\mathrm{b}, *}$, Harrie de Swart ${ }^{\mathrm{c}}$ \\ ${ }^{a}$ Institut für Informatik, Universität Kiel \\ Olshausenstraße 40, 24098 Kiel, Germany \\ ${ }^{b}$ Centre d'Economie de la Sorbonne, CNRS - Université Paris I Panthéon-Sorbonne \\ 106-112 Bd de l'Hôpital, 75647 Paris Cedex 13, France \\ ${ }^{c}$ Department of Philosophy, Tilburg University \\ P.O. Box 90153, 5000 LE Tilburg, The Netherlands
}

\begin{abstract}
Simple games are a powerful tool to analyze decision-making and coalition formation in social and political life. In this paper, we present relation-algebraic models of simple games and develop relational specifications for solving some basic problems of them. In particular, we test certain fundamental properties of simple games and compute specific players and coalitions. We also apply relation algebra to determine power indices. This leads to relation-algebraic specifications, which can be evaluated with the help of the BDD-based tool ReLVIEW after a simple translation into the tool's programming language. In order to demonstrate the visualization facilities of RELVIEW we consider an example of the Catalonian Parliament after the 2003 election.
\end{abstract}

Keywords: Relation algebra, ReLVIEw, simple game, winning coalition, swinger, dominant player, central player, power index

\section{Introduction}

A simple game is a cooperative game in which only two types of coalitions can be formed, winning coalitions and losing ones. A winning coalition takes it all while a losing coalition receives nothing. Since winning seems to be the essence of politics, simple games are extremely suitable for analyzing political situations. Important concepts in the theory of simple games are swingers, veto-players, dictators and dummies. A swinger of a winning coalition is a member of the coalition whose removal makes it losing. A veto-player is a player who is in every minimal (wrt. set inclusion) winning coalition. Under the monotonicity assumption no coalition can win without a veto-player. If one player forms the only minimal winning coalition, then it is a dictator. Consequently, a dictator is always a veto-player. There is obviously an essential difference between a veto-player and a dictator. A dictator can enforce any decision without help of the other players. In contrast, a veto-player is needed to win, but cannot win on his own. A dummy is a player who is a member of no minimal winning coalition, i.e., powerless. An important class of simple games are weighted majority games,

\footnotetext{
${ }^{2}$ Co-operation for this paper is supported by European Science Foundation EUROCORES Programme - LogICCC. We want to thank the anonymous referees for reading carefully earlier versions of this paper and for pointing out very useful comments that helped us to improve the paper.

${ }^{*}$ Corresponding author: Tel.: 0033144078 285, Fax: 0033144078 301, Email: agnieszka.rusinowska@ univ-paris1.fr 
where a weight is assigned to each player indicating its voting strength in that game. A coalition is winning if and only if the sum of the weights of its members is not smaller than a certain quota. Consider a voting body consisting of three parties $A, B$ and $C$, each having 4, 2 and 1 seats, respectively. The quota is 4 seats. Winning coalitions are the ones containing party $A$ - the only swinger of each winning coalition. Since $\{A\}$ is the only minimal winning coalition, $A$ is a dictator, and $B$ and $C$ are dummies. Suppose now that party $A$ has 3 seats. The total number of seats is 6 and the quota remains 4. There are two minimal winning coalitions $\{A, B\}$ and $\{A, C\}$, and hence $A$ is a veto-player, but it is not a dictator anymore. There are no dummies. Each member of a minimal winning coalition is a swinger, and $A$ is the only swinger of $\{A, B, C\}$.

When studying coalition formation, one of the most important issues is to identify some key players. Besides dictators and veto players several other such specific players exist in the literature. In Peleg (1981, [33]) a theory of coalition formation in simple games with dominant players has been developed. Roughly speaking, a dominant player is a player who holds a strict majority within a winning coalition. A dominant player wants to be a member of a winning coalition, in which he is dominating. For that reason he is called 'office seeking', not or hardly interested in policy. Such players neither must exist nor must be unique. Another key player is the central player introduced in Einy (1985, [18]). A central player can form a winning coalition both with the players to the 'left' of it and with the players to the 'right' of it. Such a player will be 'policy oriented'. There exists at most one central player in a simple game. In order to find it, the players must be ordered on a relevant policy dimension, and the particular position of the central player makes him very powerful. An empirical analysis of the importance and effect of dominant and central parties on cabinets in Western multiparty democracies has been examined e.g., in van Roozendaal (1992a, 1992b, 1993, 1997, [37, 38, 39, 40]). In van Roozendaal (1997, [40]) the author argues that there are certain theoretical reasons by which governments including dominant parties should be more stable than governments without dominant parties and shows, by analyzing government survival in 12 countries between 1945 and 1989, that such an effect indeed exists in real politics.

One of the most important elements of simple games, that can be applied to all kinds of organizations (e.g., political bodies, international economic organizations and business settings), is to measure the power of players. To this end, power indices have been proposed, e.g., the Shapley-Shubik index (Shapley and Shubik 1954, [43]), the Banzhaf index (Banzhaf 1965, [2]), the Deegan-Packel index (Deegan and Packel 1978, [14]), the Johnston index (Johnston 1978, [25]) and the Holler-Packel index (Holler 1982, [22]; Holler and Packel 1983, [23]). They are based on different models for power and, therefore, their use and informative value depend on the context in which they are applied. Axiomatic characterizations, as, e.g., presented in Dubey (1975, [16]), Dubey and Shapley (1979, [17]), Lehrer (1988, [28]), Laruelle and Valenciano (2001, [27]), Lorenzo-Freire et al. (2007, [30]) and Alonso-Meijide et al. (2008, [1]), are helpful for the appraisal of their applicability.

Even simple problems on simple games (like the computation of all dummy players) are in practice frequently too complicated to be solved by hand. Therefore, it is useful to have supporting computer programs available. One usually does not immediately think of programs based on relation-algebraic formulations of the concepts in question. But experience with related problems, viz. the formation of alliances and coalitions in Berghammer et al. $(2007,2009$, [8, 9]) and the computation of the strength of agents in social networks in Berghammer et al. (2010, [10]), led to the idea to apply relation algebra and a tool for its mechanization also to computational problems on simple games. In the just cited papers we combine relational algebra and RELVIEw, a computer system for the visualization and manipulation of relations and for relational prototyping and programming. In Berghammer et 
al. (2007, [8]) the relation-algebraic approach is applied to a model of stable governments, where a stable government is by definition not dominated by any other government. We formulate the notions of feasibility, dominance, and stability in relation-algebraic terms, which enables us to use RELVIEW to compute the set of all feasible stable governments. To illustrate the power of the approach, we analyze the real structure of the Polish government after the 2001 elections. It may happen that all governments are dominated. In Berghammer et al. (2009, [9]) we deal with this case by combining notions from relational algebra, graph theory and social choice theory to choose a government that is as close as possible to being un-dominated. The procedure can be executed using RELVIEW which allows to deal with graph sizes that are sufficient for practical applications. In Berghammer et al. (2010, [10]) we use relation algebra and ReLVIEw to measure power, success, and influence of an agent in a social network and to determine followers of a coalition and the kernel of an influence function. As an example the Dutch parliament is considered. Among the advantages of ReLVIEw are, for instance, short and concise programs which frequently consist of only a few lines expressing the relation-algebraic specification of the notions in question. The above idea was fortified by the fact that since a long time relation algebra and RELVIEW are successfully combined for solving problems on many discrete structures; see e.g., Berghammer et al. (1996, [5]), Berghammer et al. (2003, [11]), Berghammer and Milanese (2006, [7]), Berghammer and Fronk (2006, [4]), Berghammer (2009, [3]).

One of the aims of this paper is to apply relation algebra to the key concepts of simple games. Taking into account that all these concepts are important both from a theoretical and a practical point of view, the application of relation algebra, on the one hand being a mathematical formal approach and on the other hand giving an immediate access to the ReLVIEw implementation, is very useful. Because ReLVIEw has a very efficient BDD (Binary Decision Diagram) implementation of relations (see Leoniuk (2001, [29]), Berghammer et al. (2002, [6]) and Milanese (2003, [31])), it is able to deal with non-trivial simple games that appear, e.g., in practical political life. In addition, the tool has visualization facilities which are not easily found in other software tools and which are most helpful for fully comprehending difficult concepts and for understanding and testing the programs. In this paper we apply relation algebra to specify relations which immediately lead to power indices. Hence, our approach is particularly useful, because it allows to apply RELVIEW to compute power indices.

The remainder of the paper is structured as follows. In Section 2 the game-theoretic concepts that we deal with in the paper are presented. Section 3 introduces relation algebra and the relationalgebraic constructions which are used later. The core of the paper is Section 4. We start with two relation-algebraic models of simple games, show how they can be transformed into each other and present relation-algebraic specifications of basic properties of simple games. Thereby, the visualization facilities of RELVIEW are demonstrated in the case of the parliament of Catalonia after the 2003 election. Next, we give the relation-algebraic specifications for the sets of minimal winning coalitions and vulnerable winning coalitions, respectively, and the sets of dummies, vetoers, dictators and null players. Again, these notions are illustrated in the case of the Catalonian parliament using ReLVIEw. Thirdly, we specify relation-algebraically the central player and the notions around the dominant player. Here, the ReLVIEw tool enables us to illustrate the decisive relations underlying dominance for the Catalonian parliament. At the end of the section we specify the Banzhaf, the Holler-Packel and the Deegan-Packel power indices in terms of relation algebra and again demonstrate the ability of RELVIEW to compute these indices by means of our running example. Some concluding remarks are presented in Section 5. In particular, we recapitulate the benefits of our approach, describe important insights we have obtained from it concerning the efficient algorithmic solution of some game-theoretic problems and sketch their present realization. 


\section{Game-theoretic Preliminaries}

In this section we present the basic concepts of the theory of simple games that we refer to in the paper. More extensive treatments of simple games can be found, e.g., in Shapley (1962, [42]), Felsenthal and Machover (1998, [19]) and Peleg and Sudhölter (2003, [34]).

\subsection{Simple Games}

Following Peleg and Sudhölter (2003, [34]), a simple game is a pair $(N, \mathcal{W})$, where $N=\{1,2, \ldots, n\}$ denotes the set of players and $\mathcal{W}$ is a subset of the powerset $2^{N}$. Any element of $2^{N}$ is called a coalition. A coalition $S$ with $S \in \mathcal{W}$ is called winning, while those with $S \notin \mathcal{W}$ are called losing. A simple game $(N, \mathcal{W})$ is called monotone if $\mathcal{W}$ is an up-set in the order $\left(2^{N}, \subseteq\right)$, i.e., for all $S, T \in 2^{N}$ from $S \in \mathcal{W}$ and $S \subseteq T$ it follows $T \in \mathcal{W}$. A voting game is a monotone simple game $(N, \mathcal{W})$ with $\mathcal{W} \neq \emptyset$ and $\emptyset \notin W$. The latter two axioms exclude trivial games. A simple game is proper if the complement of a winning coalition is always losing, and strong if the complement of any losing coalition is winning. A simple game is decisive if it is both proper and strong. In the context of voting games, for instance, being proper is interesting since it is equivalent to the fact that any pair of winning coalitions has a player in common and being strong is interesting since here no blocking coalitions can occur, i.e., coalitions $S$ such that $S$ and its complement $\bar{S}$ are losing.

An important class of games are weighted majority games. They are omnipresent, in particular, if groups (commissions, boards, ... ) have to come to decisions and the members have unequal power. Usually, a weighted majority game with $n$ players is represented by a $n+1$-tuple $\left[q ; w_{1}, w_{2}, \ldots, w_{n}\right]$, where $q \in \mathbb{N}$ denotes the quota needed for a coalition to win, and $w_{k} \in \mathbb{N}$ is the weight assigned to player $k \in N$. By $w(S)=\sum_{k \in S} w_{k}$ we define the weight of a coalition $S$. A coalition $S$ is then winning if its weight is at least as large as $q$, that is, $S \in \mathcal{W}$ if and only if $w(S) \geq q$.

\subsection{Minimal Winning Coalitions and Related Notions}

Von Neumann and Morgenstern (1944, [32]) introduced the concept of a minimal winning coalition of a simple game $(N, \mathcal{W})$, that is a coalition $S \in \mathcal{W}$ such that $T \notin \mathcal{W}$ for all coalitions $T \subset S$. Less restrictive is the notion of a vulnerable winning coalition $S$. Here, besides $S \in \mathcal{W}$, it is demanded that there exists a player $k \in S$ such that $T \notin \mathcal{W}$ for all $T \subseteq S \backslash\{k\}$. In the case of a monotone game the latter property is equivalent to the existence of $k \in S$ such that $S \backslash\{k\} \notin \mathcal{W}$. Such a player $k \in S$ is called a swinger (or critical player) of $S$. These concepts are, e.g., of importance when measuring the power of players.

Apart from swingers, one can distinguish other specific players in a simple game, depending on their relation to minimal winning coalitions. Let $(N, \mathcal{W})$ be a simple game and $k \in N$. Then $k$ is called a dummy if it does not belong to a minimal winning coalition, a vetoer if it is a member of each minimal winning coalition and a dictator if $\{k\}$ is the only minimal winning coalition. Finally, $k$ is a null player if for each coalition $S \in 2^{N}$ it holds $S \cup\{k\} \in \mathcal{W}$ if and only if $S \in \mathcal{W}$.

\subsection{Central and Dominant Players}

As already mentioned in the introduction, the concept of a central player has been introduced in Einy $(1985,[18])$. Here it is assumed that the players of the game under consideration are ordered with respect to their policy positions. In political science one usually uses a left-to-right spectrum and the most important case is that the parties are ordered according to their stands in social and economic matters. 
Given a simple game $(N, \mathcal{W})$ and a policy order of the players in the form of a linear strict order $<$ on $N^{1}$, player $k \in N$ is said to be central if the connected coalition $\{j \in N: j<k\}$ to the 'left' of $k$ as well as the connected coalition $\{j \in N: k<j\}$ to the 'right' of $k$ are not winning, but both can be turned into winning coalitions when $k$ joins them.

Based on two desirability-relationships between coalitions, in Peleg (1981, [33]) the concept of dominance and dominant players is developed. Let $(N, \mathcal{W})$ be a simple game, $S, T \in 2^{N}$ be coalitions and $k \in N$ be a player. Then $S$ is called at least as desirable as $T$, written as $S \geq_{D} T$, if for all $U \in 2^{N}$ from $U \cap S=\emptyset, U \cap T=\emptyset$ and $U \cup T \in \mathcal{W}$ it follows $U \cup S \in \mathcal{W} . S$ is said to be more desirable than $T$, written as $S>_{D} T$, if $S \geq_{D} T$ but not $T \geq_{D} S$. Finally, $k$ dominates $S$, written as $k \gg S$, if $k \in S$ and $\{k\}>_{D} S \backslash\{k\}$, and $k$ is dominant if there exists a $S \in \mathcal{W}$ such that $k \gg S$. If $k$ dominates $S$, then $k$ can form a winning coalition with players outside of $S$ while $S \backslash\{k\}$ is not able to do this. The dominant players are the most powerful players of the game. Such players neither must exist nor must be unique. However, Peleg proved that in weak simple games and weighted majority games at most one dominant player may occur. Games with dominant players are called dominated.

\subsection{Power Indices}

In this section we recapitulate power indices that we deal with in the paper. One of the main power indices that can be found in the literature is the Banzhaf index (Banzhaf, 1965, [2]). Let a monotone simple game $(N, \mathcal{W})$ and a player $k \in N$ be given. Then the absolute Banzhaf index $B_{a}(k)$ of $k$ and the normalized Banzhaf index $B(k)$ of $k$ are defined as follows, where $n$ is the number of players:

$$
B_{a}(k):=\frac{\mid\{S \in \mathcal{W} \mid k \text { swinger of } S\} \mid}{2^{n-1}} \quad B(k):=\frac{B_{a}(k)}{\sum_{j \in N} B_{a}(j)}
$$

Another well-known power index that we study in the paper is the Holler-Packel index of Holler (1982, [22]) and Holler and Packel (1983, [23]). Since a minimal winning coalition $S$ coincides with the set of its swingers, the absolute Holler-Packel index $H_{a}(k)$ of $k$ can be specified in a way very similar to the definition of $B_{a}(k)$ in (1). Compared with the definition of $B_{a}(k)$, only in the numerator the set $\mathcal{W}$ is to be replaced by the set $\mathcal{W}_{\text {min }}$ of minimal winning coalitions and the denominator is to be changed to $\left|W_{\min }\right|$. The definition of the normalized Holler-Packel index exactly corresponds to the definition of $B$ via $B_{a}$ in (1). Hence, we have:

$$
H_{a}(k):=\frac{\mid\left\{S \in \mathcal{W}_{\min } \mid k \text { swinger of } S\right\} \mid}{\left|\mathcal{W}_{\min }\right|} \quad H(k):=\frac{H_{a}(k)}{\sum_{j \in N} H_{a}(j)}
$$

A power index that is related to minimal winning coalitions is also the Deegan-Packel index of Deegan and Packel (1978, [14]). Given a monotone simple game $(N, \mathcal{W})$ with set $\mathcal{W}_{\min }$ of minimal winning coalitions, the Deegan-Packel index $D(k)$ assigns to each player $k \in N$ the following number:

$$
D(k):=\frac{1}{\left|\mathcal{W}_{\min }\right|} \sum_{S \in \mathcal{W}_{\min }^{(k)}} \frac{1}{|S|}
$$

In (3) $\mathcal{W}_{\min }^{(k)}$ denotes the set of all minimal winning coalitions of the game which contain $k$.

\footnotetext{
${ }^{1}$ That is, a strict order for which $j<k$ or $k<j$ for all different players $j, k$.
} 


\section{Relation-algebraic Preliminaries}

All the concepts recapitulated in the last section will be specified again in Section 4, but then in terms of relation algebra. Before it will be done, we present the necessary relation-algebraic notions. For more details on relations and relation algebra, see, e.g., Schmidt and Ströhlein (1993, [41]) or Brink et al. (1997, [13]).

\subsection{Relation Algebra}

If $X$ and $Y$ are sets, then a subset $R$ of the Cartesian product $X \times Y$ is called a (binary) relation with domain $X$ and range $Y$. We denote the set (in this context also called type) of all relations with domain $X$ and range $Y$ by $[X \leftrightarrow Y]$ and write $R: X \leftrightarrow Y$ instead of $R \in[X \leftrightarrow Y]$. If $X$ and $Y$ are finite sets of size $m$ and $n$, respectively, then we may consider a relation $R: X \leftrightarrow Y$ as a Boolean matrix with $m$ rows and $n$ columns. The Boolean matrix interpretation of relations is well suited for many purposes and also used as one of the graphical representations of relations within the ReLVIEW tool. Therefore, in this paper we often use Boolean matrix terminology and notation. In particular, we speak of rows, columns and entries of relations and write $R_{x, y}$ instead of $\langle x, y\rangle \in R$ or $x R y$ to express that $x$ and $y$ are related via $R$.

In the present paper we use the following basic operations of relation algebra: $R^{\top}$ (transposition), $\bar{R}$ (complement), $R \cup S$ (union), $R \cap S$ (intersection) and $R S$ (composition). As special relations we use $\mathrm{O}$ (empty relation), $\mathrm{L}$ (universal relation) and I (identity relation). If $R$ is included in $S$ we write $R \subseteq S$ and equality of $R$ and $S$ is denoted as $R=S$. We assume the reader to be familiar with the component-wise descriptions of these notions, e.g., that, given $R: X \leftrightarrow Y, x \in X$ and $y \in Y$, it holds $R_{x, y}^{\top}$ if and only if $R_{y, x}$ and $\bar{R}_{x, y}$ if and only if $\neg R_{y, x}$.

\subsection{Modelling of Sets}

Relation algebra offers some simple and elegant ways to model subsets of a given set or, equivalently, predicates on this set. In this paper we will use vectors, is-element relations and injective mappings for this task.

A vector $v$ is a relation $v$ with $v=v \mathrm{~L}$. For a vector the range is irrelevant. Therefore, we consider in the following mostly vectors $v: X \leftrightarrow \mathbf{1}$ with a specific singleton set $\mathbf{1}:=\{\perp\}$ as range and omit in such cases the second subscript, i.e., write $v_{x}$ instead of $v_{x, \perp}$. Analogously to linear algebra we will use lower-case letters to denote vectors. A vector $v: X \leftrightarrow \mathbf{1}$ can be considered as a Boolean matrix with exactly one column, i.e., as a Boolean column vector, and represents (or: is a representation of) the subset $\left\{x \in X \mid v_{x}\right\}$ of $X$. A non-empty vector $v$ is a point if $v v^{\top} \subseteq$ I, i.e., it is injective. This means that it represents a singleton subset of its domain or an element from it if we identify a set $\{x\}$ with the element $x$. In the matrix model, hence, a point $v: X \leftrightarrow \mathbf{1}$ is a Boolean column vector in which exactly one entry is 1 .

Given $y \in Y$, with $R^{(y)}$ we denote the $y$-column of the relation $R: X \leftrightarrow Y$. That is, $R^{(y)}$ has type $[X \leftrightarrow \mathbf{1}]$ and for all $x \in X, R_{x}^{(y)}$ and $R_{x, y}$ are equivalent. To compare the columns of two relations $R$ and $S$ with the same domain $X$ and possible different ranges $Y$ and $Y^{\prime}$, we use the symmetric quotient

$$
\operatorname{syq}(R, S):=\overline{R^{\top} \bar{S}} \cap \overline{\bar{R}^{\top} S}
$$

of them. The type of $\operatorname{syq}(R, S)$ is $\left[Y \leftrightarrow Y^{\prime}\right]$, and transforming (4) into a component-wise notation we have for all $y \in Y$ and $y^{\prime} \in Y^{\prime}$ that $\operatorname{syq}(R, S)_{y, y^{\prime}}$ if and only if $R^{(y)}=S^{\left(y^{\prime}\right)}$, i.e., if and only if for all $x \in X$ the relationships $R_{x, y}$ and $S_{x, y^{\prime}}$ are equivalent. 
As a second way to deal with sets we will apply the relation-level equivalents of the set-theoretic symbol $\in$, that is, is-element relations $\mathrm{E}: X \leftrightarrow 2^{X}$ between $X$ and its powerset $2^{X}$. These specific relations are defined by demanding for all elements $x \in X$ and sets $Y \in 2^{X}$ that $\mathrm{E}_{x, Y}$ if and only if $x \in Y$. A simple Boolean matrix implementation of is-element relations requires an exponential number of bits. However, in Leoniuk (2001, [29]) an ingenious implementation of $\mathrm{E}: X \leftrightarrow 2^{X}$ using reduced ordered binary decision diagrams (ROBDDs) is developed, where the number of BDD-vertices is linear in the size of the base set $X$. This implementation is part of RELVIEW.

Finally, we will use injective mappings for modeling sets. Given an injective function $\imath: Y \rightarrow X$ in the usual mathematical sense, we may consider $Y$ as a subset of $X$ by identifying it with its image under $l$. If $Y$ is actually a subset of $X$ and $l$ is given as a relation of type $[Y \leftrightarrow X]$ such that $l_{y, x}$ if and only if $y=x$ for all $y \in Y$ and $x \in X$, then the vector $l^{\top} \mathrm{L}: X \leftrightarrow \mathbf{1}$ represents $Y$ as a subset of $X$ in the sense above. Clearly, the transition in the other direction is also possible, i.e., the generation of a relation $\operatorname{inj}(v): Y \leftrightarrow X$ from the vector representation $v: X \leftrightarrow \mathbf{1}$ of the subset $Y$ of $X$ such that for all $y \in Y$ and $x \in X$ we have $\operatorname{inj}(v)_{y, x}$ if and only if $y=x$. We obtain $\operatorname{inj}(v)$ by removing from $\mathrm{I}: X \leftrightarrow X$ all rows which correspond to a 0 -entry in $v$. The relation $\operatorname{inj}(v)$ is an injective mapping in the relation-algebraic sense; see, e.g., Section 4.2 of Schmidt and Ströhlein (1993, [41]). A combination of such relations with is-element relations allows a column-wise representation of sets of subsets. More specifically, if the vector $v: 2^{X} \leftrightarrow \mathbf{1}$ represents a subset $\mathcal{S}$ of $2^{X}$ in the sense above, i.e., $\mathcal{S}$ equals the set $\left\{Y \in 2^{X} \mid v_{Y}\right\}$, then for all $x \in X$ and $Y \in \mathcal{S}$ we get the equivalence of $\left(\operatorname{Einj}(v)^{\top}\right)_{x, Y}$ and $x \in Y$. This means that the elements of $\mathcal{S}$ are represented precisely by the columns of the relation $M:=\mathrm{E} \operatorname{inj}(v)^{\top}: X \leftrightarrow \mathcal{S}$ since for all $Y \in \mathcal{S}$ it holds $Y=\left\{x \in X \mid M_{x}^{(Y)}\right\}$. An illustration is given in Example 4.1 .1 by Figures 1 and 2, since the relation of Figure 1 equals $E \operatorname{inj}(v)^{\top}$ with the relation $E$ and the vector $v$ (in the transposed form) given in Figure 2.

\subsection{Cartesian Products and Applications}

Given a Cartesian product $X \times Y$ of two sets $X$ and $Y$, there are the two canonical projection functions which decompose a $\operatorname{pair}^{2} u=\left\langle u_{1}, u_{2}\right\rangle$ into its first component $u_{1}$ and its second component $u_{2}$. For a relation-algebraic approach it is useful to consider instead of these functions the corresponding projection relations $\pi: X \times Y \leftrightarrow X$ and $\rho: X \times Y \leftrightarrow Y$ such that for all $u \in X \times Y, x \in X$ and $y \in Y$ we have $\pi_{u, x}$ if and only if $u_{1}=x$ and $\rho_{u, y}$ if and only if $u_{2}=y$. Projection relations enable us to specify the well-known pairing operation of functional programming relation-algebraically as follows: For relations $R: Z \leftrightarrow X$ and $S: Z \leftrightarrow Y$ we define their pairing (frequently also called fork or tupling) $[R, S]: Z \leftrightarrow X \times Y$ by

$$
[R, S]:=R \pi^{\top} \cap S \rho^{\top} .
$$

Using (5), for all $z \in Z$ and $u \in X \times Y$ a simple reflection shows that $[R, S]_{z, u}$ if and only if $R_{z, u_{1}}$ and $S_{z, u_{2}}$. As a consequence, in the case $X=Y$ the exchange relation

$$
X:=[\rho, \pi]=\rho \pi^{\top} \cap \pi \rho^{\top}
$$

of type $[X \times X \leftrightarrow X \times X]$ exchanges the components of a pair. This means that for all $u \in X \times X$ and $v \in X \times X$ the relationship $\mathrm{X}_{u, v}$ holds if and only if $u_{1}=v_{2}$ and $u_{2}=v_{1}$.

By a combination of the constructions introduced so far, a lot of the well-known operations and

\footnotetext{
${ }^{2}$ In the present paper we always denote the first component of a pair $u \in X \times Y$ by $u_{1}$ and the second component by $u_{2}$.
} 
predicates on sets can be specified as relations. In the present paper, we need the following:

$$
\begin{array}{lll}
\mathrm{M}:=\operatorname{syq}([\mathrm{E}, \mathrm{E}], \mathrm{E}) & \mathrm{R}:=\operatorname{syq}([\overline{\mathrm{I}}, \mathrm{E}], \mathrm{E}) & \mathrm{C}:=\operatorname{syq}(\mathrm{E}, \overline{\mathrm{E}}) \\
\mathrm{J}:=\operatorname{syq}(\overline{[\overline{\mathrm{E}}, \overline{\mathrm{E}}], E)} & \mathrm{A}:=\operatorname{syq}(\overline{[\overline{\mathrm{I}}, \overline{\mathrm{E}}], E)} & \mathrm{S}:=\overline{\mathrm{E}^{\top} \overline{\mathrm{E}}}
\end{array}
$$

The relations $\mathrm{M}$ and $\mathrm{J}$ have type $\left[2^{X} \times 2^{X} \leftrightarrow 2^{X}\right]$ and relation-algebraically specify set intersection and set union, respectively, since for all $\langle S, T\rangle \in 2^{X} \times 2^{X}$ and $U \in 2^{X}$ it holds $\mathrm{M}_{\langle S, T\rangle, U}$ if and only if $S \cap T=U$ and $\mathrm{J}_{\langle S, T\rangle, U}$ if and only if $S \cup T=U$. The type of $\mathrm{R}$ and $\mathrm{A}$ is $\left[X \times 2^{X} \leftrightarrow 2^{X}\right]$, and these relations specify the removal and addition of elements, respectively. The latter means that for all $\langle x, T\rangle \in X \times 2^{X}$ and $U \in 2^{X}$ it holds $\mathrm{R}_{\langle x, T\rangle, U}$ if and only if $T \backslash\{x\}=U$ and $\mathrm{A}_{\langle x, T\rangle, U}$ if and only if $T \cup\{x\}=U$. Finally, $\mathrm{C}$ and $\mathrm{S}$ have type $\left[2^{X} \leftrightarrow 2^{X}\right]$ and for all $S, T \in 2^{X}$ it holds $\mathrm{C}_{S, T}$ if and only if $T=\bar{S}$ and $\mathrm{S}_{S, T}$ if and only if $S \subseteq T$. Hence, C specifies set complementation $S \mapsto \bar{S}:=X \backslash S$ and $S$ specifies the subset order. To demonstrate how the relation-algebraic specifications of (7) formally can be developed, we consider the most complicated case of set union. Assume $\langle S, T\rangle \in 2^{X} \times 2^{X}$ and $U \in 2^{X}$. Then we have

$$
\begin{aligned}
& S \cup T=U \Longleftrightarrow \forall x \in X:(x \in S \vee x \in T) \leftrightarrow x \in U \\
& \Longleftrightarrow \forall x \in X: \neg(x \notin S \wedge x \notin T) \leftrightarrow x \in U \\
& \Longleftrightarrow \forall x \in X: \neg\left(\overline{\mathrm{E}}_{x, S} \wedge \overline{\mathrm{E}}_{x, T}\right) \leftrightarrow \mathrm{E}_{x, U}
\end{aligned}
$$

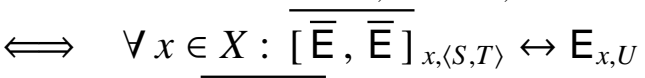

$$
\begin{aligned}
& \Longleftrightarrow \operatorname{syq}(\overline{[\overline{\mathrm{E}}, \overline{\mathrm{E}}]}, \mathrm{E})_{\langle S, T\rangle, U} \text {, }
\end{aligned}
$$

and the definition of the relation $\mathrm{J}$ in (7) shows the desired result.

We end this section with the following two functions (in the usual mathematical sense) which establish a Boolean lattice isomorphism between the two Boolean lattices $[X \leftrightarrow Y]$ and $[X \times Y \leftrightarrow \mathbf{1}]$. In the following equations $\pi: X \times Y \leftrightarrow X$ and $\rho: X \times Y \leftrightarrow Y$ are the projection relations of the underlying Cartesian product and $\mathrm{L}$ is a universal vector of type $[Y \leftrightarrow \mathbf{1}]$.

$$
\operatorname{vec}(R)=(\pi R \cap \rho) \mathrm{L} \quad \operatorname{rel}(v)=\pi^{\top}\left(\rho \cap \nu \mathrm{L}^{\top}\right)
$$

The function vec defines the vector $\operatorname{vec}(R)$ corresponding to the relation $R$, and the inverse function rel defines the relation rel $(v)$ corresponding to the vector $v$. Using a component-wise notation, these definitions say that for all $x \in X$ and $y \in Y$ we have $R_{x, y}$ if and only if $\operatorname{vec}(R)_{\langle x, y\rangle}$ and $v_{\langle x, y\rangle}$ if and only if $\operatorname{rel}(v)_{x, y}$.

\section{Investigating Simple Games with Relation Algebra}

In this section, first we introduce two relation-algebraic models of simple games and show how each of them can be transformed into the other one. Based on the vector model, we then demonstrate how to specify important notions of simple games in the language of relation algebra. All specifications can be seen as algorithms since they are either relation-algebraic expressions or inclusions respectively equations between such expressions. Hence, they can be evaluated with the help of RELVIEW after a simple translation into the programming language of this tool. 


\subsection{Relation-Algebraic Models of Simple Games}

A first possibility to model a simple game $(N, \mathcal{W})$ with relation-algebraic means is to use a vector $v: 2^{N} \leftrightarrow \mathbf{1}$ that represents the set $\mathcal{W}$ as subset of $2^{N}$ in the sense of Section 3.2. Frequently, $v$ is called the characteristic vector of the game; in our context we call it the vector model. Given such a model $v$, from Section 3.2 we already know that then the columns $M^{(S)}, S \in \mathcal{W}$, of the relation $M:=\mathrm{E} \operatorname{inj}(v)^{\top}: N \leftrightarrow \mathcal{W}$ precisely represent all winning coalitions. Hence, the game $(N, \mathcal{W})$ can also be modeled by the relation $M$. Since $M$ specifies membership of players in winning coalitions, i.e., $M_{k, S}$ if and only if $k \in S$, for all $k \in N$ and $S \in \mathcal{W}$, we call it the membership model. The definition of $M$ shows how to transform the vector model into the membership model. We formulate this once again as the first part of the following theorem, where $E$ is the is-element relation between players and coalitions. In the second part of the theorem we show how to obtain the vector model back from the membership model.

Theorem 4.1.1. Let $(N, \mathcal{W})$ be a simple game. If $v: 2^{N} \leftrightarrow \mathbf{1}$ is the game's vector model, then $\mathrm{E} \operatorname{inj}(v)^{\top}: N \leftrightarrow \mathcal{W}$ is its membership model. Conversely, if $M: N \leftrightarrow \mathcal{W}$ is the game's membership model, then syq(E, $M) \mathrm{L}: 2^{N} \leftrightarrow \mathbf{1}$ (with $\mathrm{L}: \mathcal{W} \leftrightarrow \mathbf{1}$ ) is its vector model.

Proof: Due to the above remark, we only have to show that if $M$ is the membership model then $\operatorname{syq}(\mathrm{E}, M)$ is the vector model. For all $S \in 2^{N}$ we get

$$
\begin{aligned}
(\operatorname{syq}(\mathrm{E}, M) \mathrm{L})_{S} & \Longleftrightarrow \exists T \in \mathcal{W}: \operatorname{syq}(\mathrm{E}, M)_{S, T} \wedge \mathrm{L}_{T} \\
& \Longleftrightarrow \exists T \in \mathcal{W}: \forall k \in N: \mathrm{E}_{k, S} \leftrightarrow M_{k, T} \\
& \Longleftrightarrow \exists T \in \mathcal{W}: \forall k \in N: k \in S \leftrightarrow k \in T \\
& \Longleftrightarrow \exists T \in \mathcal{W}: S=T \\
& \Longleftrightarrow S \in \mathcal{W} .
\end{aligned}
$$

This property shows that the vector syq $(\mathrm{E}, M) \mathrm{L}$ represents $\mathcal{W}$ as subset of $2^{N}$, as required for the vector model of the game.

Choosing one of the two relation-algebraic models to specify a game-theoretic notion usually depends on the analyzed concept. Since the columns of the membership model $M$ of $(N, \mathcal{W})$ enumerate the winning coalitions and since it can hardly be seen from the vector model $v$ which coalitions are winning, the relation $M$ is more appropriate if one wants RELVIEW to compute the winning coalitions. However, as experience has shown, the great advantage of the vector model is that it enables in many cases much more elegant relation-algebraic specifications than the membership model. This holds in particular if a task requires to treat coalitions which are non-winning. For $S \in 2^{N}$ in the vector model the property $S \notin \mathcal{W}$ is simply expressed by $\bar{v}_{S}$, whereas in the membership model, for instance, it may require to consider the vector representation $s: N \leftrightarrow \mathbf{1}$ of $S$ and to verify $\operatorname{syq}(M, s)=0$. Specifications that are based on the vector model are frequently even more efficient than membershipbased ones. This is especially the case if a high percentage of coalitions is winning, since then in the membership model a lot of columns occur. That almost half of the coalitions are winning is typical in practice. E.g., using data from van Deemen (1989, [15]), van Roozendaal (1990, [36]) and Berghammer et al. (2010, [10]), with the help of ReLVIEw we obtained for Dutch parliaments that from the 8192 possible coalitions of the 13-parties parliament after the 1972 election 3999 (48.8\%) are winning, from the 1024 possible coalitions of the present 10-parties parliament $505(49.3 \%)$ are winning and from the 64 possible coalitions of the 6-parties parliament after the 1986 election even 32 
(50\%) are winning. Apart from the visualization of input and output, in the remainder of the paper we restrict ourselves to the vector model. In the next theorem we give first examples for relation-algebraic specifications of game-theoretic notions that are based on the vector model. In it, $S: 2^{N} \leftrightarrow 2^{N}$ denotes the subset order as introduced in (7).

Theorem 4.1.2. Assume $v: 2^{N} \leftrightarrow \mathbf{1}$ to be the vector model of a simple game $(N, \mathcal{W})$. Then $(N, \mathcal{W})$ is monotone if and only if $\mathrm{S} \bar{v} \subseteq \bar{v}$ and is a voting game if and only if in addition $v \neq \mathrm{O}$ and $v \subseteq \mathrm{E}^{\top} \mathrm{L}$.

Proof: That $S \bar{v} \subseteq \bar{v}$ specifies monotonicity follows from

$$
\begin{aligned}
S \bar{v} \subseteq \bar{v} & \Longleftrightarrow \forall S \in 2^{N}:(S \bar{v})_{S} \rightarrow \bar{v}_{S} \\
& \Longleftrightarrow \forall S \in 2^{N}:\left(\exists T \in 2^{N}: S_{S, T} \wedge \bar{v}_{T}\right) \rightarrow \bar{v}_{S} \\
& \Longleftrightarrow \forall S, T \in 2^{N}: S \subseteq T \wedge T \notin \mathcal{W} \rightarrow S \notin \mathcal{W} \\
& \Longleftrightarrow \forall S, T \in 2^{N}: S \subseteq T \wedge S \in \mathcal{W} \rightarrow T \in \mathcal{W} .
\end{aligned}
$$

The equivalence of $v \neq O$ and $\mathcal{W} \neq \emptyset$ is trivial and the remaining claim is shown by

$$
\begin{aligned}
v \subseteq \mathrm{E}^{\top} \mathrm{L} & \Longleftrightarrow \forall S \in 2^{N}: v_{S} \rightarrow \exists k \in N: \mathrm{E}_{S, k}^{\top} \wedge \mathrm{L}_{k} \\
& \Longleftrightarrow \forall S \in 2^{N}: S \in \mathcal{W} \rightarrow \exists k \in N: k \in S \\
& \Longleftrightarrow \forall S \in 2^{N}: S \in \mathcal{W} \rightarrow S \neq \emptyset .
\end{aligned}
$$

In the next theorem we specify relation-algebraically the properties of a simple game of being proper and strong. Here C : $2^{N} \leftrightarrow 2^{N}$ is the relation for set complementation; cf. (7).

Theorem 4.1.3. Given $v: 2^{N} \leftrightarrow 1$ as the vector model of a simple game $(N, \mathcal{W})$, the game is proper if and only if $v \subseteq \mathrm{C} \bar{v}$ and the game is strong if and only if $\bar{v} \subseteq \mathrm{C} v$.

Proof: Starting with a formal logical specification of being a proper game, the first claim is shown by

$$
\begin{aligned}
\forall S \in 2^{N}: S \in \mathcal{W} \rightarrow \bar{S} \notin \mathcal{W} & \Longleftrightarrow \forall S \in 2^{N}: S \in \mathcal{W} \rightarrow \exists T \in 2^{N}: T=\bar{S} \wedge T \notin \mathcal{W} \\
& \Longleftrightarrow \forall S \in 2^{N}: v_{S} \rightarrow \exists T \in 2^{N}: \mathrm{C}_{S, T} \wedge \bar{v}_{T} \\
& \Longleftrightarrow v \subseteq \mathrm{C} \bar{v} .
\end{aligned}
$$

In the same way the second specification can be calculated.

Since $C$ is a mapping in the relation-algebraic sense, we get due to Schmidt and Ströhlein (1993, [41]) that $\bar{v} \subseteq \mathrm{C} v$ if and only if $\mathrm{C} \bar{v}=\overline{\mathrm{C} v} \subseteq v$. Hence, the simple game is decisive (i.e., proper and strong) if and only if $v=\mathrm{C} \bar{v}$ if and only if $\bar{v}=\mathrm{C} v$.

Also for weighted majority games a vector model $v: 2^{N} \leftrightarrow \mathbf{1}$ can be computed within relation algebra. To this end, the players are interpreted as the parties of a parliament and the weights are interpreted as the number of the parliament seats the party holds, i.e., in the very same way as in real political life. Then the only requirement to obtain $v$ is that, given $X$ as set of seats, there is a mapping (in the relation-algebraic sense) $D: X \leftrightarrow N$ at hand that describes the distribution of the seats, i.e., fulfills for all $x \in X$ and $k \in N$ that $D_{x, k}$ if and only if seat $x$ is owned by party $k$. Since the concrete procedure is irrelevant for the remainder of the paper, we do not go into details here and refer the interested reader to Berghammer et al. (2010, [10]), where the computation of $v$ from $D$ formally is developed. 


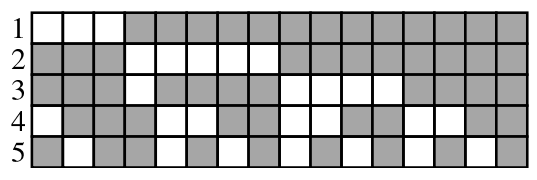

Figure 1: Membership model of the Catalonian game

In general, the number of winning coalitions of a simple game can grow rapidly with the number of players. For example, if the game is proper and strong and $n$ is the number of players, then the number of winning coalitions is $2^{n-1}$, i.e., $50 \%$ of all coalitions are winning. Therefore, in the following example that shall demonstrate the visualization facilities of RELVIEw we deal with a rather small game, taken from Lorenzo-Freire et al. (2007, [30]).

Example 4.1.1. We consider the following weighted majority game with five players, that is a representation of the parliament of Catalonia, one of the 17 Spanish autonomous communities, after the November 2003 election.

$$
[68 ; 46,42,23,15,9]
$$

The players are, from left to right, labeled with the numbers 1, 2, 3, 4 and 5; they correspond (in the same order) to the five Catalonian parties CIU, PSC-CPC, ERC, PP and ICV-EA. In the picture of Figure 1 the membership model $M: N \leftrightarrow \mathcal{W}$ of this game is shown as depicted by ReLVIEW in the relation-window of its user interface. In this $5 \times 16$ Boolean matrix a black square means a 1-entry and a white square means a 0 -entry. So, for example, the winning coalition represented by the first column of $M$ consists of the three parties PSC-CPC, ERC and ICV-EA. If we transform the membership model $M$ into the vector model, we obtain a vector $v: 2^{N} \leftrightarrow \mathbf{1}$ in which exactly 16 entries are 1 . The two pictures of Figure 2 show the is-element relation $\mathrm{E}: N \leftrightarrow 2^{N}$ and, below it, the transpose of the vector $v$ (that is, the row vector $v^{\top}: \mathbf{1} \leftrightarrow 2^{N}$ ). The 32 columns of the is-element relation $\mathrm{E}$ represent the 32 coalitions. A comparison of the pictures (here the row vector representation of the game is of great advantage) shows that the 1-entries of the vector model $v$ precisely designate those columns of $\mathrm{E}$ that belong to the membership model $M$.

As a weighted majority game, $(N, \mathcal{W})$ is monotone. We have also tested whether it is proper and strong using the RELVIEW-versions

$$
\operatorname{proper}(\mathrm{E}, \mathrm{v})=\operatorname{incl}(\mathrm{v}, \operatorname{Compl}(\mathrm{E}) *-\mathrm{v}) \quad \operatorname{strong}(\mathrm{E}, \mathrm{v})=\operatorname{incl}(-\mathrm{v}, \operatorname{Compl}(\mathrm{E}) * \mathrm{v})
$$

of the relation-algebraic specifications of Theorem 4.1.3, where the pre-defined RELVIEW-operation incl tests inclusion of relations and the RELVIEW-function

$$
\operatorname{Compl}(E)=\operatorname{syq}(-E,-E)
$$

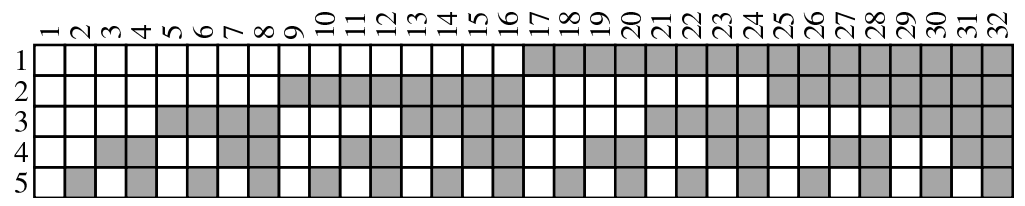

Figure 2: Vector model of the Catalonian game 
computes from the is-element relation $\mathrm{E}: N \leftrightarrow 2^{N}$ the relation $\mathrm{S}: 2^{N} \leftrightarrow 2^{N}$ for set complementation. In both cases we obtained the answer 'yes'.

\subsection{Computing Minimal Coalitions and Related Notions}

Computing minimal winning coalitions with relation-algebraic means is easy. It is well-known, cf. Schmidt and Ströhlein (1993, [41]) that, given a strict order relation $R: X \leftrightarrow X$ and a vector $w: X \leftrightarrow \mathbf{1}$ that represents a subset $Y$ of $X$, the vector $w \cap \overline{R^{\top} w}: X \leftrightarrow \mathbf{1}$ represents the set of minimal elements of $Y$ as a subset of $X$. Hence, if we take $w$ as vector model $v: 2^{N} \leftrightarrow \mathbf{1}$ of a simple game $(N, \mathcal{W})$ and $R$ as the irreflexive part of the subset order $S: 2^{N} \leftrightarrow 2^{N}$, we get immediately the following result.

Theorem 4.2.1. If $v: 2^{N} \leftrightarrow \mathbf{1}$ is the vector model of the simple game $(N, \mathcal{W})$, then the vector

$$
\operatorname{minwin}(v):=v \cap \overline{(\mathrm{S} \cap \overline{\mathrm{l}})^{\top} v}
$$

of type $\left[2^{N} \leftrightarrow 1\right]$ represents the set $\mathcal{W}_{\min }$ of minimal winning coalitions.

Next, we specify relation-algebraically the is-swinger relation and the vector of vulnerable winning coalitions. To simplify the calculations, we only consider monotone games. With regard to practical applications this is no serious restriction. ${ }^{3}$ Recall from Section 3, that $R$ is the relation-algebraic specification of element-removal and the function rel yields for a vector that represents a subset of a Cartesian product in the sense of Section 3.2 the corresponding 'proper' relation.

Theorem 4.2.2. Let $v: 2^{N} \leftrightarrow \mathbf{1}$ be the vector model of a monotone simple game $(N, \mathcal{W})$. If we define the is-swinger relation Swingers $(v): N \leftrightarrow 2^{N}$ and the vector vulwin( $(v): 2^{N} \leftrightarrow \mathbf{1}$ by

$$
\operatorname{Swingers}(v):=\mathrm{E} \cap \mathrm{L}^{\top} \cap \operatorname{rel}(\mathrm{R} \bar{v}) \quad \operatorname{vulwin}(v):=\operatorname{Swingers}(v)^{\top} \mathrm{L}
$$

(with $\mathrm{L}: N \leftrightarrow \mathbf{1}$ ), then for all $k \in N$ and $S \in 2^{N}$ it holds $\operatorname{Swingers}(v)_{k, S}$ if and only if $k$ is a swinger of $S$ and vulwin $(v)_{S}$ if and only if $S$ is a vulnerable winning coalition.

Proof: For all $k \in N$ and $S \in 2^{N}$ we have

$$
\begin{aligned}
\text { Swingers }(v)_{k, S} & \Longleftrightarrow\left(\mathrm{E} \cap \mathrm{L} v^{\top} \cap \operatorname{rel}(\mathrm{R} \bar{v})\right)_{k, S} \\
& \Longleftrightarrow \mathrm{E}_{k, S} \wedge\left(\mathrm{L} v^{\top}\right)_{k, S} \wedge \operatorname{rel}(\mathrm{R} \bar{v})_{k, S} \\
& \Longleftrightarrow \mathrm{E}_{k, S} \wedge\left(\mathrm{L}^{\top}\right)_{k, S} \wedge(\mathrm{R} \bar{v})_{\langle k, S\rangle} \\
& \Longleftrightarrow \mathrm{E}_{k, S} \wedge\left(\mathrm{L} v^{\top}\right)_{k, S} \wedge \exists T \in 2^{N}: \mathrm{R}_{\langle k, S\rangle, T} \wedge \bar{v}_{T} \\
& \Longleftrightarrow \mathrm{E}_{k, S} \wedge v_{S} \wedge \exists T \in 2^{N}: S \backslash\{k\}=T \wedge \bar{v}_{T} \\
& \Longleftrightarrow \mathrm{E}_{k, S} \wedge v_{S} \wedge \bar{v}_{S \backslash\{k\}} \\
& \Longleftrightarrow k \in S \wedge S \in \mathcal{W} \wedge S \backslash\{k\} \notin \mathcal{W} .
\end{aligned}
$$

\footnotetext{
${ }^{3}$ In the political science literature typically one only considers monotone simple games as, e.g., in Peleg (1981, [33]), or even demands a simple game to be monotone by definition as, e.g., van Deemen (1989, [15]) and van Roozendaal (1990, [36]) do.
} 
Since $(N, \mathcal{W})$ is monotone, the last formula specifies $k$ as a swinger of $S$. This is the first result. Using it, the second one is shown by

$$
\begin{aligned}
\operatorname{vulwin}(v)_{S} & \Longleftrightarrow\left(\text { Swingers }(v)^{\top} \mathrm{L}\right)_{S} \\
& \Longleftrightarrow \exists k \in N: \operatorname{Swingers}(v)_{S, k}^{\top} \wedge \mathrm{L}_{k} \\
& \Longleftrightarrow \exists k \in N: k \in S \wedge S \in \mathcal{W} \wedge S \backslash\{k\} \notin \mathcal{W} \\
& \Longleftrightarrow S \in \mathcal{W} \wedge \exists k \in N: k \in S \wedge S \backslash\{k\} \notin \mathcal{W} .
\end{aligned}
$$

So far, we have considered swingers and specific coalitions. In the remainder of the section, we turn towards specific players with more or less power such as a dummy, a vetoer, a dictator and a null player. The next theorem shows how the sets of these specific players can be specified relationalgebraically as vectors. It uses the relation A of (7) for the addition of an element.

Theorem 4.2.3. Based on the vector model $v: 2^{N} \leftrightarrow 1$ of a simple game and $m:=\operatorname{minwin}(v)$ as vector representation of the set $\mathcal{W}_{\min }$ of minimal winning coalitions, we consider the following four vectors of type $[N \leftrightarrow \mathbf{1}]$ (where $[N \leftrightarrow N]$ is the type of the I in $\operatorname{syq}(\mathrm{I}, \mathrm{E})$ and $\left[2^{N} \leftrightarrow 2^{N}\right]$ is the type of the I in $\overline{\mathrm{I} m}$ ):

$$
\begin{aligned}
\operatorname{dummy}(m) & :=\overline{\mathrm{E} m} & \operatorname{vetoer}(m) & :=\overline{\overline{\mathrm{E}}} m \\
\operatorname{dictator}(m) & :=\operatorname{syq}(\mathrm{I}, \mathrm{E})(m \cap \overline{\overline{\mathrm{I}} m}) & \operatorname{null}(v) & :=\operatorname{syq}\left(\operatorname{rel}(\mathrm{A} v)^{\top}, v\right)
\end{aligned}
$$

Then dummy $(m)$ (vetoer $(m)$, dictator $(m)$ and null $(v)$, respectively) represents the set of dummies (vetoers, dictators and null players, respectively).

Proof: We only verify the specifications for dictators and null players. Assume $k \in N$. Then the first case follows from

$$
\begin{aligned}
\operatorname{dictator}(m)_{k} & \Longleftrightarrow(\operatorname{syq}(\mathrm{I}, \mathrm{E})(m \cap \overline{\bar{I} m}))_{k} \\
& \Longleftrightarrow \exists S \in 2^{N}: \operatorname{syq}(\mathrm{I}, \mathrm{E})_{k, S} \wedge m_{S} \wedge \overline{\bar{I}}_{S} \\
& \Longleftrightarrow \exists S \in 2^{N}:\left(\forall j \in N: \mathrm{I}_{j, k} \leftrightarrow \mathrm{E}_{j, S}\right) \wedge m_{S} \wedge \neg \exists T \in \mathcal{W}: \bar{I}_{S, T} \wedge m_{T} \\
& \Longleftrightarrow \exists S \in 2^{N}:(\forall j \in N: j=k \leftrightarrow j \in S) \wedge m_{S} \wedge \forall T \in \mathcal{W}: m_{T} \rightarrow S=T \\
& \Longleftrightarrow \exists S \in 2^{N}: S=\{k\} \wedge S \in \mathcal{W}_{\min } \wedge \forall T \in \mathcal{W}_{\min }: S=T
\end{aligned}
$$

and the second case follows from

$$
\begin{aligned}
\operatorname{null}(v)_{k} & \Longleftrightarrow \operatorname{syq}\left(\operatorname{rel}(A v)^{\top}, v\right)_{k} \\
& \Longleftrightarrow \forall S \in 2^{N}: \operatorname{rel}(A v)_{S, k}^{\top} \leftrightarrow v_{S} \\
& \Longleftrightarrow \forall S \in 2^{N}:(A v)_{\langle k, S\rangle} \leftrightarrow v_{S} \\
& \Longleftrightarrow \forall S \in 2^{N}:\left(\exists T \in 2^{N}: A_{\langle k, S\rangle, T} \wedge v_{T}\right) \leftrightarrow v_{S} \\
& \Longleftrightarrow \forall S \in 2^{N}:\left(\exists T \in 2^{N}: S \cup\{k\}=T \wedge T \in \mathcal{W}\right) \leftrightarrow S \in \mathcal{W} \\
& \Longleftrightarrow \forall S \in 2^{N}: S \cup\{k\} \in \mathcal{W} \leftrightarrow S \in \mathcal{W},
\end{aligned}
$$

since in both cases the last formula of the derivation is the formal logical specification of the property under consideration. 


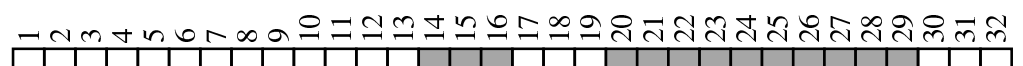

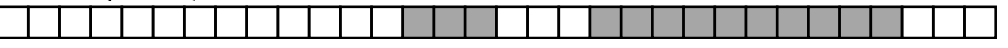

Figure 3: Vulnerable and minimal winning coalitions of the Catalonian game

Let us consider what the RELVIEW-programs corresponding to the above specifications yield in the case of our running example. Since the is-swinger relation is decisive for computing power indices, we postpone its picture until Section 4.4 that is devoted to this topic.

Example 4.2.1. If the RELVIEW-programs we have obtained from the relation-algebraic specifications vulwin( $(v)$ and $\operatorname{minwin}(v)$ of Theorem 4.2.2 and Theorem 4.2.1 are applied to the vector model of Example 4.1.1, then the tool yields two vectors which, again in transposed form to save space, are shown in the two pictures of Figure 3. The row vector on the top designates the 13 vulnerable winning coalitions of the parliament of Catalonia after the 2003 election, and that under it designates the five minimal winning coalitions. From these results we could obtain the 'concrete' form of the coalitions by a comparison with the columns of the is-element relation $\mathrm{E}: N \leftrightarrow 2^{N}$ as remarked in Example 4.1.1. The much more easier way is, however, to use the technique for the column-wise enumeration of sets presented in Section 3.2, i.e., to evaluate the two expressions $\mathrm{E}$ vulwin $(v)^{\top}$ and $\mathrm{E} \operatorname{minwin}(v)^{\top}$. Doing so, we obtain the left-most and right-most of the three RELVIEW-matrices of Figure 4, from which each vulnerable winning coalition and each minimal winning coalition, respectively, can immediately be obtained as a column. The RELVIEW-matrix in the middle column-wisely enumerates the sets of swingers of the vulnerable winning coalitions. It is obtained by removing from the is-swinger relation all columns corresponding to a 0 -entry in the vector representation of the vulnerable winning coalitions. Relation-algebraically this reads as $\operatorname{Swingers}(v)$ inj(vulwin $(v))^{\top}$.

To explain the three RELVIEW-matrices of Figure 4 a bit more, we compare the first columns of the two $5 \times 13$ matrices. Since they are identical, that means, each party is a swinger, the represented coalition $\{$ PSC-CPC, ERC, ICV-EA\} is a minimal winning one. This agrees with the column-wise enumeration of these coalitions in which the coalition appears, too. Next, we compare the third columns of the two matrices. From the first matrix we get $\{$ PSC-CPC, ERC, PP, ICV-EA $\}$ as vulnerable winning coalition and from the second one $\{P S C-C P C, E R C\}$ as the set of its swingers. Hence, this coalition is not minimal winning. Again this agrees with the right-most matrix, since now the coalition does not occur as a column.

To demonstrate ReLVIEw's visualization potential a bit more, the ReLVIEW-graph of Figure 5 shows the Hasse-diagram of the inclusion order $\mathrm{S}$ of the 32 coalitions of our example. In this picture the inclusion relationships between the 16 winning coalitions are highlightened by boldface arcs; from this it becomes immediately clear that the game is monotone. The five minimal winning coalitions are drawn as white squares and the 11 non-minimal winning coalitions are drawn as black circles.

For our running example we also have computed the vectors specified in Theorem 4.2.3. Here all results delivered by the RELVIEW tool were empty.
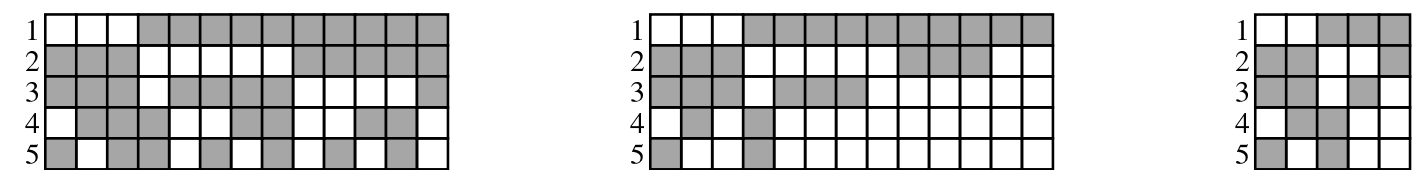

Figure 4: Column-wise enumeration of the vulnerable and minimal winning coalitions 


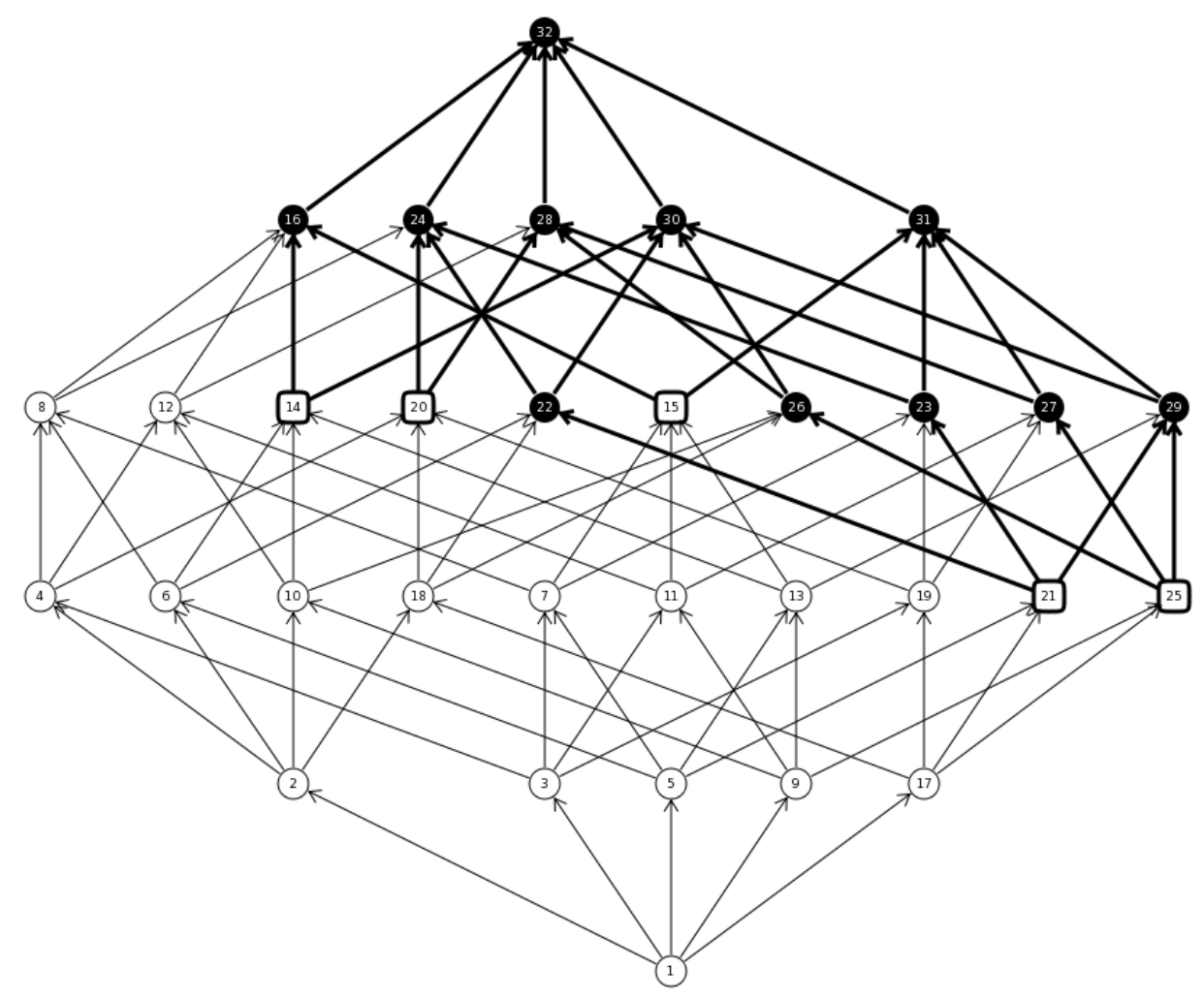

Figure 5: The ordered coalitions of the Catalonian game

\subsection{Computing Central and Dominant Players}

In this section we deliver relation-algebraic specifications of the sets of central and dominant players. Let us start with the concept of a central player. Note that since there exists at most one central player in a simple game, the vector given in the following theorem either is empty or is a point in the sense of Section 3.2. Since the vector is specified as relation-algebraic expression, we use the letter $P$ for the policy order and not the infix-symbol < as in Section 2.3.

Theorem 4.3.1. Let a simple game $(N, \mathcal{W})$ with a linear strict policy order $P: N \leftrightarrow N$ be given and assume that $v: 2^{N} \leftrightarrow \mathbf{1}$ is the game's vector model. Then the vector

$$
\operatorname{central}(v, P):=\overline{\operatorname{syq}(P, \mathrm{E}) v} \cap \overline{\operatorname{syq}\left(P^{\top}, \mathrm{E}\right) v} \cap \operatorname{syq}(P \cup \mathrm{I}, \mathrm{E}) v \cap \operatorname{syq}\left(P^{\top} \cup \mathrm{I}, \mathrm{E}\right) v
$$

of type $[N \leftrightarrow \mathbf{1}]$ (where I : $N \leftrightarrow N$ ) represents the set of central players.

Proof: Let $k \in N$ be a player. Then we have

$$
\begin{aligned}
(\overline{\operatorname{syq}(P, \mathrm{E}) v})_{k} & \Longleftrightarrow \neg \exists S \in 2^{N}: \operatorname{syq}(P, \mathrm{E})_{k, S} \wedge v_{S} \\
& \Longleftrightarrow \forall S \in 2^{N}:\left(\forall j \in N: P_{j, k} \leftrightarrow \mathrm{E}_{j, S}\right) \rightarrow \bar{v}_{S} \\
& \Longleftrightarrow \forall S \in 2^{N}:\left(\forall j \in N: P_{j, k} \leftrightarrow j \in S\right) \rightarrow \bar{v}_{S} \\
& \Longleftrightarrow \forall S \in 2^{N}: S=\left\{j \in N: P_{j, k}\right\} \rightarrow S \notin \mathcal{W} \\
& \Longleftrightarrow\left\{j \in N: P_{j, k}\right\} \notin \mathcal{W}
\end{aligned}
$$


and a replacement of $P$ by its transpose in this calculation shows

$$
\overline{\operatorname{syq}\left(P^{\top}, \mathrm{E}\right) v} \Longleftrightarrow\left\{j \in N: P_{k, j}\right\} \notin \mathcal{W} .
$$

Next, we deal with the third expression of the intersection and get

$$
\begin{aligned}
(\operatorname{syq}(P \cup \mathrm{I}, \mathrm{E}) v)_{k} & \Longleftrightarrow \exists S \in 2^{N}: \operatorname{syq}(P \cup \mathrm{I}, \mathrm{E})_{k, S} \wedge v_{S} \\
& \Longleftrightarrow \exists S \in 2^{N}:\left(\forall j \in N:\left(P_{j, k} \vee \mathrm{I}_{j, k}\right) \leftrightarrow \mathrm{E}_{j, S}\right) \wedge v_{S} \\
& \Longleftrightarrow \exists S \in 2^{N}:\left(\forall j \in N:\left(P_{j, k} \vee j=k\right) \leftrightarrow j \in S\right) \wedge v_{S} \\
& \Longleftrightarrow \exists S \in 2^{N}: S=\left\{j \in N: P_{j, k}\right\} \cup\{k\} \wedge S \in \mathcal{W} \\
& \Longleftrightarrow\left\{j \in N: P_{j, k}\right\} \cup\{k\} \in \mathcal{W} .
\end{aligned}
$$

Again by a replacement of $P$ by $P^{\top}$ we find for the fourth expression

$$
\left(\operatorname{syq}\left(P^{\top} \cup \mathrm{I}, \mathrm{E}\right) v\right)_{k} \Longleftrightarrow\left\{j \in N: P_{k, j}\right\} \cup\{k\} \in \mathcal{W} .
$$

If we read $P_{j, k}$ as $j<k$, the conjunction of the right-hand sides of the derived equivalences precisely means that $k$ is a central player.

Next, let us study the concept of a dominant player. In the following, we show how two desirability concepts introduced in Peleg (1981, [33]) can be specified relation-algebraically. We do it again in such a way that this leads to RELVIEw-programs after a simple translation step.

In the decisive first part of the following theorem it is shown how the concept 'at-least-as-desirable' can be specified relation-algebraically by means of a vector with a Cartesian product as domain. The again vector-based - specifications of 'more-desirable' and 'dominance' then are easy consequences of the theorem's first part. Recall from Section 3, that $\mathrm{J}$ and $\mathrm{R}$ are the relation-algebraic specification of set union and element-removal, respectively, $X$ is the relation for exchanging the components of pairs, the function rel transforms vector representations into 'proper' relations and the function vec is the inverse of rel.

Theorem 4.3.2. Let $v: 2^{N} \leftrightarrow \mathbf{1}$ be the vector model of a simple game $(N, \mathcal{W})$. Then the vector

$$
\operatorname{alades}(v):=\overline{\mathrm{L}\left(\left[\overline{\mathrm{E}^{\top} \mathrm{E}}, \overline{\mathrm{E}^{\top} \mathrm{E}}\right] \cap[\operatorname{rel}(\mathrm{J} \bar{v}), \operatorname{rel}(\mathrm{J} v)]\right)}
$$

of type $\left[2^{N} \times 2^{N} \leftrightarrow \mathbf{1}\right]$ (where $\mathrm{L}: \mathbf{1} \leftrightarrow 2^{N}$ ) represents the at-least-as-desirable relation $\geq_{D}$ as subset of $2^{N} \times 2^{N}$. For the more-desirable relation $>_{D}$ the same is obtained by the vector

$$
\operatorname{mdes}(v):=\operatorname{alades}(v) \cap \overline{\mathrm{X} \operatorname{alades}(v)}
$$

of type $\left[2^{N} \times 2^{N} \leftrightarrow \mathbf{1}\right]$. With $\pi: N \times 2^{N} \leftrightarrow N$ as first projection of $N \times 2^{N}$, finally, the vector

$$
\operatorname{dom}(v):=\operatorname{vec}(\mathrm{E}) \cap[\pi \operatorname{syq}(\mathrm{l}, \mathrm{E}), \mathrm{R}] \operatorname{mdes}(v)
$$

of type $\left[N \times 2^{N} \leftrightarrow 1\right]$ represents the dominance relation $\gg$ as subset of $N \times 2^{N}$. 
Proof: To prove the first claim, let a pair $\langle S, T\rangle \in 2^{N} \times 2^{N}$ be given. Then we have the following equivalence:

$$
\begin{aligned}
& \operatorname{alades}(v)_{\langle S, T\rangle} \Longleftrightarrow \overline{\mathrm{L}\left(\left[\overline{\mathrm{E}^{\top} \mathrm{E}}, \overline{\mathrm{E}^{\top} \mathrm{E}}\right] \cap[\operatorname{rel}(\mathrm{J} \bar{v}), \operatorname{rel}(\mathrm{J} v)]\right)_{\langle S, T\rangle}} \\
& \Longleftrightarrow \neg \exists U \in 2^{N}: \mathrm{L}_{\perp, U} \wedge\left[\overline{\mathrm{E}^{\top} \mathrm{E}}, \overline{\mathrm{E}^{\top} \mathrm{E}}\right]_{U,\langle S, T\rangle} \wedge[\operatorname{rel}(\mathrm{J} \bar{v}), \operatorname{rel}(\mathrm{J} v)]_{U,\langle S, T\rangle} \\
& \Longleftrightarrow \neg \exists U \in 2^{N}: \overline{\mathrm{E}}^{\top} \mathrm{E}_{U, S} \wedge{\overline{\mathrm{E}^{\top} \mathrm{E}}}_{U, T} \wedge \operatorname{rel}(\mathrm{J} \bar{v})_{U, S} \wedge \operatorname{rel}(\mathrm{J} v)_{U, T} \\
& \Longleftrightarrow \neg \exists U \in 2^{N}:{\overline{\mathrm{E}^{\top} \mathrm{E}}}_{U, S} \wedge{\overline{\mathrm{E}^{\top} \mathrm{E}}}_{U, T} \wedge(\mathrm{J} \bar{v})_{\langle U, S\rangle} \wedge(\mathrm{J} v)_{\langle U, T\rangle} \\
& \Longleftrightarrow \forall U \in 2^{N}:{\overline{\mathrm{E}^{\top} \mathrm{E}}}_{U, S} \wedge{\overline{\mathrm{E}^{\top} \mathrm{E}}}_{U, T} \wedge(\mathrm{J} v)_{\langle U, T\rangle} \rightarrow \neg(\mathrm{J} \bar{v})_{\langle U, S\rangle}
\end{aligned}
$$

Now, we consider the four relationships of the body of the quantification. We calculate

$$
\overline{\mathrm{E}}^{\top} \mathrm{E}_{U, S} \Longleftrightarrow \neg \exists j \in N: \mathrm{E}_{U, j}^{\top} \wedge \mathrm{E}_{j, S} \Longleftrightarrow \neg \exists j \in N: j \in U \wedge j \in S \Longleftrightarrow U \cap S=\emptyset
$$

for the first one. In the same way we get the equivalence of the relationship $\overline{\mathrm{E}}^{\mathrm{T}} \overline{\mathrm{E}}_{U, T}$ and $U \cap T=\emptyset$. For the third relationship we obtain

$$
\begin{aligned}
(\mathrm{J} v)_{\langle U, T\rangle} & \Longleftrightarrow \exists V \in 2^{N}: \mathrm{J}_{\langle U, T\rangle, V} \wedge v_{V} \\
& \Longleftrightarrow \exists V \in 2^{N}: U \cup T=V \wedge V \in \mathcal{W} \\
& \Longleftrightarrow U \cup T \in \mathcal{W} .
\end{aligned}
$$

A similar calculation shows that $(\mathrm{J} \bar{v})_{\langle U, S\rangle}$ if and only if $U \cup S \notin \mathcal{W}$. Summing up, we have shown the equivalence

$$
\operatorname{alades}(v)_{\langle S, T\rangle} \Longleftrightarrow \forall U \in 2^{N}: U \cap S=\emptyset \wedge U \cap T=\emptyset \wedge U \cup T \in \mathcal{W} \rightarrow U \cup S \in \mathcal{W},
$$

the right-hand side of which is the formal logical specification of the relationship $S \geq_{D} T$ and, thus, concludes the proof of the first claim.

To verify the second claim we assume again a pair $\langle S, T\rangle \in 2^{N} \times 2^{N}$ to be given. Then the desired result is shown by the following derivation, since the last line of it is the formal logical specification of $S>_{D} T$ :

$$
\begin{aligned}
\operatorname{mdes}(v)_{\langle S, T\rangle} & \Longleftrightarrow \quad \text { alades }(v) \cap \overline{\mathrm{X} \operatorname{alades}(v)})_{\langle S, T\rangle} \\
& \Longleftrightarrow \text { alades }(v)_{\langle S, T\rangle} \wedge \overline{\mathrm{X} \operatorname{alades}(v)} \\
& \left.\Longleftrightarrow S \geq_{D} T \wedge \neg\right\rangle\langle U, V\rangle \in 2^{N} \times 2^{N}: \mathrm{X}_{\langle S, T\rangle,\langle U, V\rangle} \wedge \operatorname{alades}(v)_{\langle U, V\rangle} \\
& \Longleftrightarrow S \geq_{D} T \wedge \neg \exists\langle U, V\rangle \in 2^{N} \times 2^{N}: S=V \wedge T=U \wedge \operatorname{alades}(v)_{\langle U, V\rangle} \\
& \Longleftrightarrow S \geq_{D} T \wedge \neg \operatorname{alades}(v)_{\langle T, S\rangle} \\
& \Longleftrightarrow S \geq_{D} T \wedge \neg\left(T \geq_{D} S\right)
\end{aligned}
$$

Finally, the last claim is shown by the following calculation for all pairs $\langle k, S\rangle \in N \times 2^{N}$, which uses the equivalence of $\operatorname{syq}(\mathrm{I}, \mathrm{E})_{k, T}$ and $\{k\}=T$ and ends with the logical formula that specifies the 


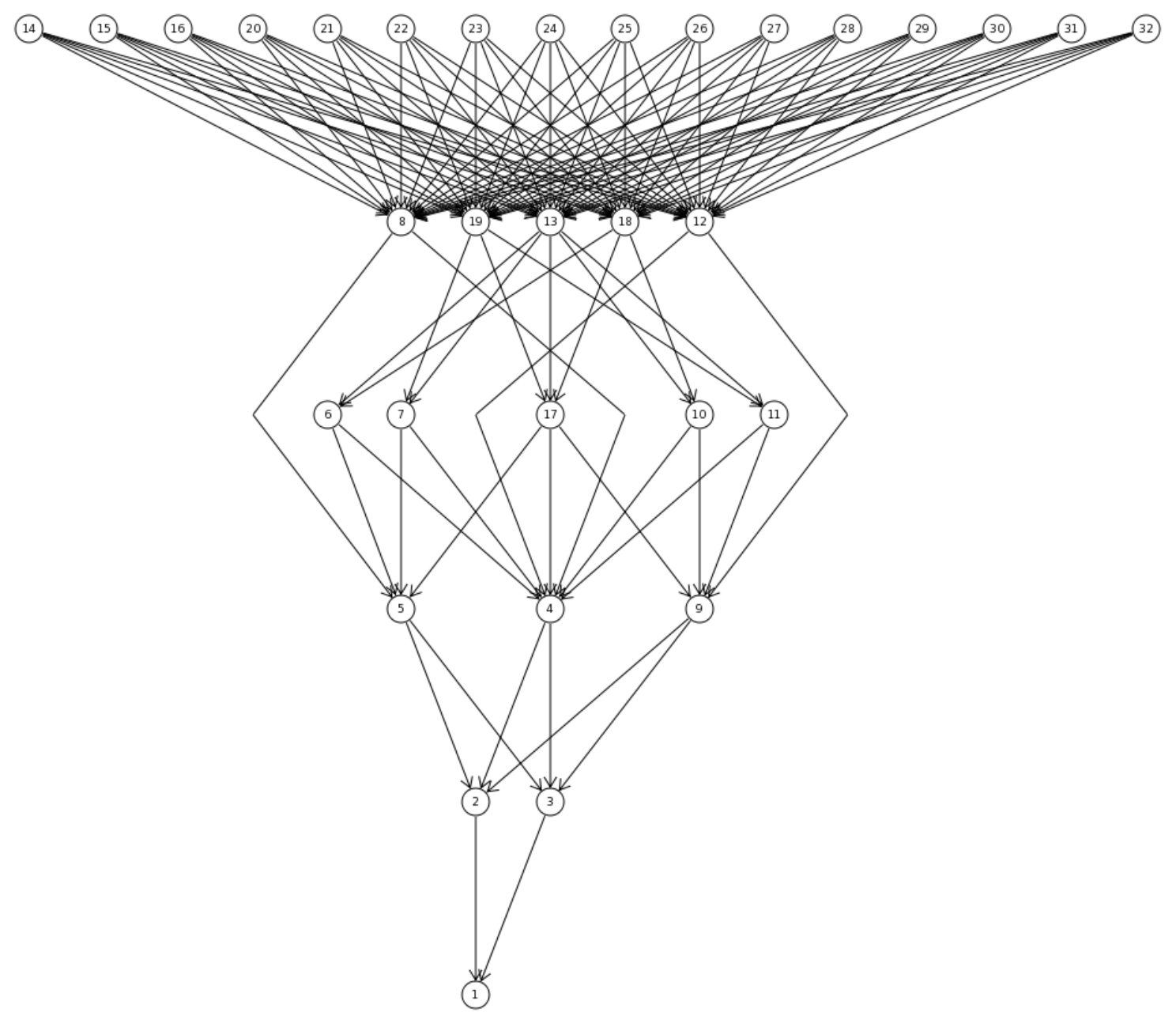

Figure 6: The more-desirable relation of the Catalonian game

relationship $k \gg S$ :

$$
\begin{aligned}
\operatorname{dom}(v)_{\langle k, S\rangle} & \Longleftrightarrow(\operatorname{vec}(\mathrm{E}) \cap[\pi \operatorname{syq}(\mathrm{I}, \mathrm{E}), \mathrm{R}] \operatorname{mdes}(v))_{\langle k, S\rangle} \\
& \Longleftrightarrow \operatorname{vec}(\mathrm{E})_{\langle k, S\rangle} \wedge([\pi \operatorname{syq}(\mathrm{I}, \mathrm{E}), \mathrm{R}] \operatorname{mdes}(v))_{\langle k, S\rangle} \\
& \Longleftrightarrow \mathrm{E}_{k, S} \wedge \exists\langle T, U\rangle \in 2^{N} \times 2^{N}:\left[\pi \operatorname{syq}(\mathrm{I}, \mathrm{E}), \mathrm{R}_{\langle k, S\rangle,\langle T, U\rangle} \wedge \operatorname{mdes}(v)_{\langle T, U\rangle}\right. \\
& \Longleftrightarrow k \in S \wedge \exists\langle T, U\rangle \in 2^{N} \times 2^{N}:\left(\operatorname{syq}(\mathrm{I}, \mathrm{E})_{k, T} \wedge \mathrm{R}_{\langle k, S\rangle, U} \wedge T>_{D} U\right. \\
& \Longleftrightarrow k \in S \wedge \exists\langle T, U\rangle \in 2^{N} \times 2^{N}:\{k\}=T \wedge S \backslash\{k\}=U \wedge T>_{D} U \\
& \Longleftrightarrow k \in S \wedge\{k\}>_{D} S \backslash\{k\}
\end{aligned}
$$

If we apply the function rel of (8) to the three vectors of Theorem 4.3.2, then we obtain again relationalgebraic specifications $\operatorname{rel}(\operatorname{alades}(v)), \operatorname{rel}(\operatorname{mdes}(v))$ and $\operatorname{rel}(\operatorname{dom}(v))$ for the relations $\geq_{D},>_{D}$ and $\gg$, respectively, but now as 'proper' relations of type $\left[2^{N} \leftrightarrow 2^{N}\right]$ in the first two cases and $\left[N \leftrightarrow 2^{N}\right]$ in the latter case. The RELVIEw-versions of $\operatorname{rel}(\operatorname{alades}(v))$ and $\operatorname{rel}(\operatorname{mdes}(v))$ allow to visualize the at-least-asdesirable and the more-desirable relation of a simple game $(N, \mathcal{W})$ with vector model $v: 2^{N} \leftrightarrow \mathbf{1}$ not only as Boolean matrices but also as directed graphs. As we have already demonstrated, in the latter case additionally features are provided which allow to draw graphs nicely and to highlight selected 


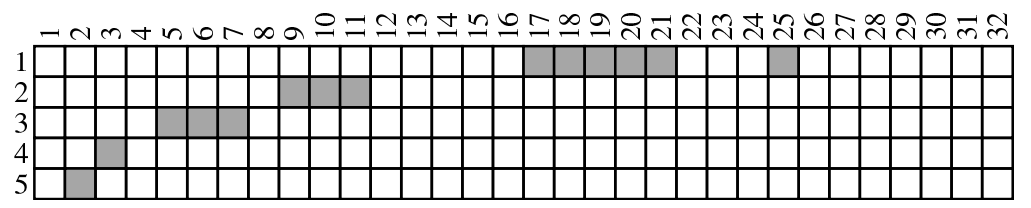

Figure 7: The dominance relation of the Catalonian game

portions. The specification $\operatorname{rel}(\operatorname{dom}(v))$ at once leads to a RELVIEW-program for determining the game's dominant players. Recall that the dominant players are those which are related to an element of $\mathcal{W}$ via the dominance relation $\gg$. Taking $v$ as vector representation of $\mathcal{W}$ and $\operatorname{rel}(\operatorname{dom}(v))$ as relationalgebraic specification of $\gg$, this immediately yields $\operatorname{rel}(\operatorname{dom}(v)) v: N \leftrightarrow \mathbf{1}$ as vector representation of the set of dominant players of the game.

Example 4.3.1. For our running example now we demonstrate how RELVIEW can be used to treat the concepts of 'desirability' and 'dominance'. In the RELVIEw-picture of Figure 6 we show the Hassediagram of the more-desirable relation $\operatorname{rel}(\operatorname{mdes}(v))$ of the parliament of Catalonia after the 2003 election. The directed graph is drawn using the level-oriented graph-drawing algorithm of Gansner et al. (1993, [21]). The meaning of the arrow is that one coalition is more desirable than another one. From the level at the top we get that half of the coalitions is maximal with respect to 'moredesirability' and these coalitions coincide with the winning ones (since the row vector representation of the winning coalitions of Example 4.1.1 says that precisely the columns of $\mathrm{E}$ with labels 14-16 or labels 20-32 represent winning coalitions). In the case of the dominance-relation $\operatorname{rel}(\operatorname{dom}(v))$ : $N \leftrightarrow 2^{N}$, the RELVIEw tool delivers the $5 \times 32$ Boolean matrix of Figure 7. It shows that party CIU (row number 1 in the matrix) is the only dominant player of the parliament because it dominates the three winning coalitions with column labels 20, 21 and 25. Recall from the ReLVIEw-picture of the vector model in Example 4.1.1 that the coalitions with column labels 17-19 and the coalitions dominated by the other parties are not winning.

\subsection{Computing Power Indices}

In this section we apply relation algebra to some power indices. More precisely, we present relation-algebraic specifications that immediately lead to the Banzhaf, Holler-Packel and DeeganPackel indices. There is a very close relationship between the relation Swingers $(v): N \leftrightarrow 2^{N}$ of Theorem 4.2.2 and the power indices introduced in (1) and (2) that also is the key for their computation using the ReLVIEW tool. This relationship is presented in the next theorem. To enhance readability, for $X$ and $Y$ being finite, $R: X \leftrightarrow Y$ and $x \in X$, we denote the number of 1-entries of $R$ by $|R|$ and the number of 1-entries of the $x$-row of $R$ by $|R|_{x}$. Hence, $|R|$ equals the cardinality of $R$ (as set of pairs) and $|R|_{x}$ equals the cardinality of the subset $Y^{\prime}$ of $Y$ that is represented by the transpose of the $x$-row in the sense of Section 3.2.

Theorem 4.4.1. Assume a monotone simple game $(N, \mathcal{W})$ with $n$ players and its vector model $v$ : $2^{N} \leftrightarrow \mathbf{1}$. Furthermore, let a player $k \in N$ be given. Then we have for the Banzhaf index that

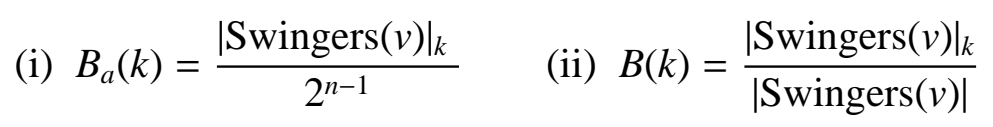

and for the Holler-Packel index that

(iii) $H_{a}(k)=\frac{\mid \text { Swingers }\left.(\min w i n(v))\right|_{k}}{|\min w i n(v)|}$

(iv) $H(k)=\frac{\mid \text { Swingers }\left.(\operatorname{minwin}(v))\right|_{k}}{|\operatorname{Swingers}(\min w i n(v))|}$. 


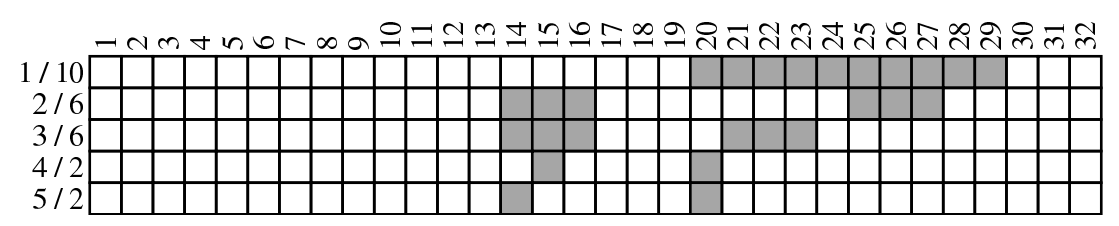

Figure 8: The is-swinger relation of the Catalonian game

Proof: Equation (i) is trivial since the transpose of the $k$-row of Swingers $(v)$ represents the set $\{S \in$ $\mathcal{W} \mid k$ swinger of $S\}$. Combining it with the definition of $B(k)$, we get

$$
B(k)=\frac{B_{a}(k)}{\sum_{j \in N} B_{a}(j)}=\frac{\frac{1}{2^{n-1}}|\operatorname{Swingers}(v)|_{k}}{\frac{1}{2^{n-1}} \sum_{j \in N}|\operatorname{Swingers}(v)|_{j}}=\frac{|\operatorname{Swingers}(v)|_{k}}{|\operatorname{Swingers}(v)|}
$$

which is (ii). Equation (iii) is again trivial and (iv) is shown analogously to (ii).

If the RELVIEw tool depicts a relation $R$ as Boolean matrix in the relation-window, then in the window's status bar the number of 1-entries of $R$ is shown. Furthermore, it is able to mark its rows and columns for explanatory purposes. So far, we have only shown the possibility to attach consecutive row and/or column numbers. But also the numbers of 1-entries can be attached as labels. In combination with Theorem 4.4.1 this immediately allows to compute Banzhaf and Holler-Packel indices. We demonstrate this by means of our running example.

Example 4.4.1. If we use ReLVIEW to compute the is-swinger relation Swingers(v) for the vector model $v$ of our running Catalonia parliament example and additionally instruct the tool to attach consecutive row and column numbers, and for each row also the number of its 1-entries as second label (after the sign '/'), we get the picture of Figure 8. From the second row labels 10, 6, 6, 2, 2 and the fact that there are exactly 26 1-entries, we immediately obtain the following normalized Banzhaf indices of the parties:

$$
\text { CIU: } \frac{10}{26} \quad \text { PSC-CPC: } \frac{6}{26} \quad \text { ERC: } \frac{6}{26} \quad \text { PP: } \frac{2}{26} \quad \text { ICV-EA: } \frac{2}{26}
$$

If in these fractions the denominators 26 are changed to $2^{5-1}=16$, then the results are the parties, absolute Banzhaf indices (in the same order) $\frac{10}{16}, \frac{6}{16}, \frac{6}{16}, \frac{2}{16}$ and $\frac{2}{16}$. Next, we evaluate the expression Swingers(minwin(v)). Then RELVIEW depicts the labeled Boolean matrix of Figure 9 on its screen. Hence, the normalized Holler-Packel indices of the Catalonian parties are as follows:

$$
\text { CIU: } \frac{3}{13} \quad \text { PSC-CPC: } \frac{3}{13} \quad \text { ERC: } \frac{3}{13} \quad P P: \frac{2}{13} \quad \text { ICV-EA }: \frac{2}{13}
$$

In Example 4.2.1 we have shown that there are five minimal winning coalitions. As a consequence, a change of the denominators 13 to 5 yields the absolute Holler-Packel indices of the parties as (in the same order) $\frac{3}{5}, \frac{3}{5}, \frac{3}{5}, \frac{2}{5}$ and $\frac{2}{5}$.

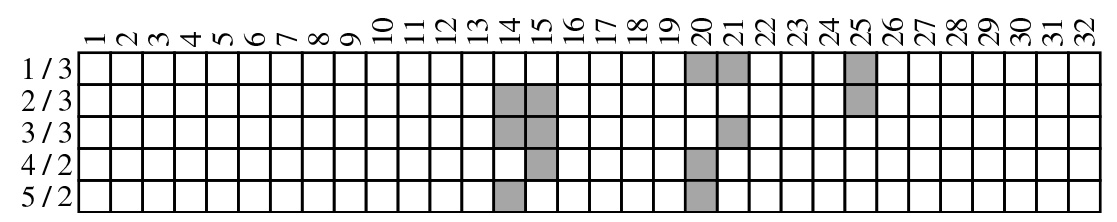

Figure 9: The is-swinger relation wrt. the minimal winning coalitions 

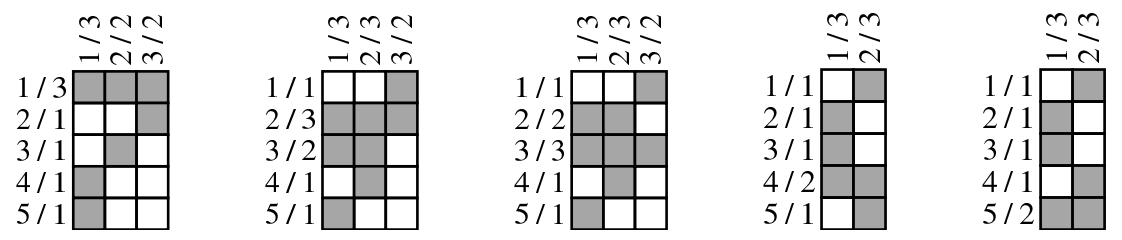

Figure 10: Relations for determining the Deegan-Packel indices

The Shapley-Shubik index, the Deegan-Packel index and the Johnston index are three further prominent power indices for measuring power in simple games. In contrast with the Banzhaf and HollerPackel indices, their definitions use more arithmetic operations than (1) and (2). As we will show in the next example by means of the Deegan-Packel index, in principle relation algebra and RELVIEW can also be applied here. But the example also shows the limit of the use of RELVIEW in respect thereof.

Example 4.4.2. To compute the Deegan-Packel index $D(k)$ of player $k \in N$ using relation algebra and the ReLVIEW tool, we assume the vector representation $m:=\operatorname{minwin}(v)$ of the set $W_{\min }$ of minimal winning coalitions to be at hand and the player $k \in N$ to be represented by a point $p: N \leftrightarrow \mathbf{1}$ in the sense of Section 3.2. If $\mathrm{E}: N \leftrightarrow 2^{N}$ is the is-element relation, then a little reflection shows that the vector $\mathrm{E}^{\top} p: 2^{N} \leftrightarrow \mathbf{1}$ represents the set of all coalitions $S \in 2^{N}$ such that $k \in S$, and hence the relation

$$
\operatorname{Deegan}(m, p):=\operatorname{Einj}\left(m \cap \mathrm{E}^{\top} p\right)^{\top}
$$

of type $\left[N \leftrightarrow \mathcal{W}_{\min }^{(k)}\right]$ column-wisely represents the set $\mathcal{W}_{\min }^{(k)}$ used in (3) to define $D(k)$. Based on $m$ and (9), now $D(k)$ can be determined by performing one after another the following three steps:

1. Compute for each column of Deegan $(m, p)$ the reciprocal value of the number of its 1-entries.

2. Add all numbers obtained by the first step.

3. Divide the result of the second step by the number of 1-entries of $m$.

In the case of our Catalonian parliament example, the RELVIEW tool delivered the five relations $\operatorname{Deegan}(m, p)$ which are depicted in Figure 10, where the point $p$ represents (from left to right) the five parties CIU, PSC-CPC, ERC, PP and ICV-EA. If we apply the above procedure, then we obtain from the second column labels of these matrices that $D(C I U)=\frac{1}{5}\left(\frac{1}{3}+\frac{1}{2}+\frac{1}{2}\right)=\frac{8}{30}, D(P S C-C P C)=$ $\frac{1}{5}\left(\frac{1}{3}+\frac{1}{3}+\frac{1}{2}\right)=\frac{7}{30}, D(E R C)=\frac{1}{5}\left(\frac{1}{3}+\frac{1}{3}+\frac{1}{2}\right)=\frac{7}{30}, D(P P)=\frac{1}{5}\left(\frac{1}{3}+\frac{1}{3}\right)=\frac{4}{30}$, and $D(I C V-E A)=\frac{1}{5}\left(\frac{1}{3}+\frac{1}{3}\right)=\frac{4}{30}$.

It is obvious that the calculations of Example 4.4.2 can hardly be done by hand if the number of minimal winning coalitions is large. For instance, the situation becomes a good deal worse in the case of the present 10-parties Dutch parliament, since here already 42 of the 505 winning coalitions are minimal winning. To overcome the difficulties caused by the restrictive programming language of ReLVIEw ${ }^{4}$ the Kure library has been developed; see Milanese (2003, [31]), Szymanski (2003, [44]). It comprises the core functionality of RELVIEW and opens the possibility to integrate relation-algebraic computations into C- and Java-programs. Particularly with regard to the above example, a use of KURE allows to perform all the arithmetic computations we have done by hand automatically by the superordinate C or Java-program.

\footnotetext{
${ }^{4}$ Caused by the specific application domain of the tool, relations are the only pre-defined datatype of this language and all further datatypes have to be modeled via them. In particular, real numbers and their base operations do not exist and it seems to be very difficult to model the reals in the same elegant and efficient way as, e.g., sets and a lot of structures of discrete mathematics and computer science.
} 


\section{Concluding Remarks}

In this paper we have presented two relation-algebraic models of simple games. For the vector model, we have developed relation-algebraic specifications for testing fundamental properties of simple games and for computing specific players and coalitions, and some relations which are important for determining dominance and power indices. All specifications are algorithmic and can be evaluated by the BDD-based tool ReLVIEW after a straightforward translation into the tool's programming language. To demonstrate the visualization facilities of ReLVIEW, we have used the Catalonian Parliament after the 2003 election as example.

The correctness of all relation-algebraic specifications we have presented and, hence, also of the corresponding RELVIEW-programs is guaranteed by the extremely formal calculations that drastically reduce the danger of making errors. In fact, we have obtained the results by developing formally the relation-algebraic expressions and formulas from the original predicate-logic specifications. The formulation in the prevalent mathematical theorem-proof-style has only been chosen to emphasize the results and to enhance readability. We regard this goal-oriented development of programs from formal specifications that are correct by construction as the first advantage of our approach. As the second advantage of our approach we regard its computer-support by means of an appropriate tool. All relation-algebraic specifications we have developed are expressed by extremely short and concise RELVIEW-programs. Consequently, these are easy to alter in the case of slightly changed specifications, e.g., if winning coalitions additionally have to be connected (i.e., to form intervals w.r.t. a given policy order) as in Einy $(1985$, [18]) or to be feasible w.r.t. a set of policies in the sense of Berghammer et al. (2007, [8]) or if, as in van Deemen (1989, [15]), in the definition of $S \geq_{D} T$ only for all non-empty $U \in 2^{N}$ from $U \cap S=\emptyset, U \cap T=\emptyset$ and $U \cup T \in \mathcal{W}$ it has to follow that $U \cup S \in \mathcal{W}$. Combining this feature of RELVIEW with its possibilities for visualization and stepwise execution of programs allows the user to experiment and play with established as well as new concepts while avoiding unnecessary expenditure of work. This makes the tool very useful for scientific research. Nowadays, systematic experiments are accepted as a way for obtaining new insights and scientific results, and tools for this purpose become increasingly important as one proceeds in investigations. The very efficient ReLVIEW implementation of relations via BDDs was of immense help for the problems we have treated in this paper. Due to it, without any problems we have been able to apply our algorithms to a lot of simple games originating from real political life. Such simple games are presented, e.g., in Peleg (1981, [33]), van Deemen (1989, [15]), van Roozendaal (1990, [36]) and Freixas and Molinero (2009, [20]).

Many problems appearing in connection with simple games are known to be intractable in terms of complexity theory, for instance, \#P-complete ${ }^{5}$ or NP-hard. See e.g., Prasad and Kelly (1990, [35]) for more details. There are special algorithms tailored for such hard problems, like the $O\left(n \cdot 2^{\frac{n}{2}}\right)$ algorithm for the Banzhaf index and the $O\left(n^{2} \cdot 2^{\frac{n}{2}}\right)$ algorithm for the Shapley-Shubik index of weighted majority games presented in Klinz and Woeginger (2005, [26]), and the multilinear-extension-based algorithms mentioned in Alonso-Meijide et al. (2008, [1]) and Lorenzo-Freire et al. (2007, [30]). In spite of the fact that RELVIEW implements relations very efficiently, frequently it cannot compete with these special algorithms. To give an example, with the programs resulting from the present paper we have not been able to tackle the United States Federal System game with 537 players that is described in Freixas and Molinero (2009, [20]).

\footnotetext{
${ }^{5}$ This complexity class was introduced in Vailant (1979, [45]) to characterize the number of solutions to an NPcomplete decision problem.
} 
It is impossible to estimate the largest $n$ for which RELVIEW can reasonably perform calculations for simple games, because this very much depends on the relation one wants to evaluate, on the number of minimal winning coalitions and other things. But ReLVIEW has dealt with many examples from real life with up till 20 players in a very efficient way.

In RELVIEW it is possible to indicate for a relation $R$ of its workspace the number of vertices of the BDD implementing $R$. By the experiments performed with the tool we have noticed that in almost all cases the numbers of vertices of the BDDs for the vector of winning coalitions are relatively small and the same is true for the BDDs of the results of the computations. E.g., the BDD of the vector model of the 13-parties Dutch parliament after the 1972 election consists of 13 vertices only and 17 BDDvertices suffice to implement the is-swinger relation of this game. If problems appeared in the form of very large BDDs, then those typically implemented intermediate results during the computations and were then caused by modifications of BDDs for which the use of the relation-algebraic operations proved to be unnecessarily complicated. As a consequence, we came to the insight that BDDs seem to be an excellent means for solving game-theoretic problems efficiently if they are manipulable in full generality and not only via the relation-algebraic operations (the programming language of RELVIEW, respectively). In the following, we briefly sketch our present work in this regard.

Due to RELVIEW's particular implementation of relations using BDDs, relational vectors with $2^{n}$ rows as introduced in Section 3.2 correspond exactly to Boolean functions with $n$ input variables that are implemented by BDDs (see Leoniuk (2001, [29]) and Berghammer et al. (2002, [6]). So, if we represent the set of winning coalitions $\mathcal{W}$ of a simple game with $n$ players by a relational vector in ReLVIEw (or the KuRE library mentioned above), it internally uses a BDD with $n$ variables to implement the characteristic function of $\mathcal{W}$. This representation of the set of winning coalitions of a simple game using a BDD is the starting point of Bolus (2010, [12]). In this paper, besides the representation size of (vector-)weighted majority games, also the computation of the minimal winning coalitions and the computation of some common power indices is investigated. Due to the relationship between relational vectors and BDDs, the results are directly transferable and usable in the context of RELVIEw. For instance, a BDD — and thus a vector model in our sense — of a weighted majority game with quota $q$ and $n$ players can be built in expected worst-case running time $O(n \cdot q)$ and the resulting BDD has size at most $n(q+1)$. Provided constant time arithmetic on integer and rational numbers, Banzhaf and Holler-Packel indices of all players can be computed in expected worst case running time $O(n \cdot q)$ and the Shapley-Shubik and Deegan-Packel indices of all players can be computed in expected worst case running time $O\left(n^{3} \cdot q\right)$, if the players are ordered by non-increasing weights. In Bolus (2010, [12]) results for vector-weighted games and the general case are presented for most of the four power indices. In the BDDs framework one can also apply, e.g., the upper bound of $O\left(2^{\frac{n}{2}}\right)$ to the size of the BDD of any weighted majority game; see Hosaka et al. (1994, [24]).

But, in contrast to the purely relation-algebraic approach of the present paper, the development of game-theoretic algorithms based on BDDs requires much more effort, the implementation has to be much more elaborated, and it often requires further knowledge of more technical issues such as complementary edges. Moreover, the correctness proofs are much more complicated and much less formal than ours - with all negative consequences. Additionally, using relation algebra as "intermediate language" does not produce less efficient algorithms in general. To the contrary, many algorithms are usable in practice and are reasonably fast. If they are not adequate for the problem domain, the implementation of relations often allows to change perspective and to utilize BDDs directly without friction as seen here. This way, ReLVIEw can be seen as a platform which incorporates not only relation algebra, but also BDDs, which are known to be applicable to a lot of computationally 
hard problems. The current direction in the development of ReLVIEW is to make it more extensible by expanding its interface in such a way that it is possible to outsource program logic into small problem-specific modules, so-called plug-ins. By specific game-theoretic plug-ins that are based on Bolus (2010, [12]), in the meantime we are able to deal also with large problems that cannot be solved with the original ReLVIEw tool, such as the above mentioned United States Federal System game with 537 players. To give an impression of concrete running times, for this example the algorithm of Bolus (2010, [12]) computes a BDD for the set of winning coalitions that has about 67000 vertices and, from it, then the Banzhaf indices of all players - altogether in less than 1 second. But it is most important that the experiments with ReLVIEw led to this new BDD-approach to game-theoretic problems. It should additionally be remarked that the experiments frequently even gave the decisive hints how the algorithms have to work.

In Bolus (2010, [12]) only a portion of the problems we have solved in this paper is investigated. For the future, we plan to extend this approach also to the remaining problems, i.e., to implement corresponding plug-ins for ReLVIEW. First experiments in respect thereof led to the impression that testing fundamental properties and determining key players directly by means of the BDD-representation of $\mathcal{W}$ should not be very difficult. But we fear that computing the relations decisive for dominance from this BDD remains as difficult as in the case of the relation-algebraic approach.

\section{References}

[1] Alonso-Meijide, J.M., Casas-Mendez, B., Holler, M.J., Lorenzo-Freire, S., 2008. Computing power indices: Multilinear extensions and new characterizations. European Journal of Operational Research 188, 540-554.

[2] Banzhaf, J.F., 1965. Weighted voting doesn't work: A mathematical analysis. Rutgers Law Review 19, $317-343$.

[3] Berghammer R., 2009. Applying relation algebra and ReLVIEW to solve problems on orders and lattices. Acta Informatica 45, 211-236.

[4] Berghammer R., Fronk A., 2006. Exact computation of minimum feedback vertex sets with relational algebra. Fundamenta informatica 70, 301-316.

[5] Berghammer, R., Karger von, B., Ulke, C., 1996. Relation-algebraic analysis of Petri nets with ReLVIEw, In: Margaria, T., Steffen, B. (Eds.), Proc. 2nd Workshop "Tools and Applications for the Construction and Analysis of Systems", LNCS 1055, Springer, 49-69.

[6] Berghammer, R., Leoniuk, B., Milanese, U., 2002. Implementation of relational algebra using binary decision diagrams, In: de Swart, H. (Ed.), Proc. 6th Workshop "Relational Methods in Computer Science", LNCS 2561, Springer, 241-257.

[7] Berghammer, R., Milanese, U., 2006. Relational approach to Boolean logic problems. In: McGaull et al, (Eds.), Proc. 8th Workshop "Relational Methods in Computer Science", LNCS 3929, Springer, 48-59.

[8] Berghammer, R., Rusinowska, A., de Swart, H., 2007. Applying relation algebra and ReLVIEw to coalition formation. European Journal of Operational Research 178, 530-542.

[9] Berghammer, R., Rusinowska, A., de Swart, H., 2009. An interdisciplinary approach to coalition formation. European Journal of Operational Research 195, 487-496.

[10] Berghammer, R., Rusinowska, A., de Swart, H., 2010. Applying relation algebra and ReLVIEw to measures in a social network. European Journal of Operational Research 202, 182-195.

[11] Berghammer, R., Schmidt, G., Winter, M., 2003. ReLVIEw and RATH - Two systems for dealing with relations, In: de Swart, H., Orlowska, E., Schmidt, G., Roubens, M. (Eds.): Theory and Applications of Relational Structures as Knowledge Instruments. LNCS 2929, Springer, 1-16.

[12] Bolus, S., 2010. Power indices of simple games and vector-weighted majority games by means of binary decision diagrams. Submitted.

[13] Brink, C., Kahl, W., Schmidt, G. (Eds.), 1997. Relational Methods in Computer Science, Advances in Computing Science, Springer.

[14] Deegan, J., Packel, F.W., 1978. A new index of power for simple $n$-person games. International Journal of Game Theory 7, 113-123. 
[15] van Deemen, A., 1989. Dominant players and minimum size coalitions. European Journal of Political Research 17, 313-332.

[16] Dubey, P., 1975. On the uniqueness of the Shapley value. International Journal of Game Theory 4, 131-139.

[17] Dubey, P., Shapley, L.S., 1979. Mathematical properties of the Banzhaf power index. Mathematics of Operations Research 4, 99-131.

[18] Einy, E., 1985. On connected coalitions in dominated simple games. International Journal of Game Theory 14 , 103-125.

[19] Felsenthal, D.S., Machover, M., 1998. The measurement of voting power: theory and practice, problems and paradoxes, London: Edward Elgar Publishers.

[20] Freixas J., Molinero X., 2009. Simple games and weighted games: A theoretical and computational viewpoint. Discrete Applied Mathematics 157, 1496-1508.

[21] Gansner, E.R., Koutsofios, E., North, S.C., Vo, K.P., 1993. A technique for drawing directed graphs, IEEE Trans. Software Eng. 19, 214-230.

[22] Holler, M.J., 1982. Forming coalitions and measuring voting power. Political Studies 30, 262-271.

[23] Holler, M.J., Packel, E.W., 1983. Power, luck and the right Index. Journal of Economics 43, 21-29.

[24] Hosaka, K., Takenaga, Y., Yajima, S., 1994. On the size of ordered binary decision diagrams representing threshold functions In: Ding-Zhu, D., Xiang-Sun, Z. (Eds.), Proc. 5th International Symphosium on Algorithms and Computation. LNCS 834, Springer, 584-592.

[25] Johnston, R.J., 1978. On the measurement of power: Some reactions to Laver. Environment and Planning A 10, 907-914.

[26] Klinz, B., Woeginger, G.J., 2005. Faster algorithms for computing power indices in weighted voting games. Mathematical Social Sciences 49, 111-116.

[27] Laruelle, A., Valenciano, F., 2001. Shapley-Shubik and Banzhaf indices revisited. Mathematics of Operations Research 26, 89-104.

[28] Lehrer, E., 1988. An axiomatization of the Banzhaf value. International Journal of Game Theory 17, 89-99.

[29] Leoniuk B., 2001. ROBDD-basierte Implementierung von Relationen und relationalen Operationen mi Anwendungen. Ph.D. thesis, Universität Kiel.

[30] Lorenzo-Freire, S., Alonso-Meijide, J.M., Casas-Mendez, B., Fiestras-Janeiro, M.G., 2007. Characterizations of the Deegan-Packel and Johnston power indices. European Journal of Operational Research 177, 431-444.

[31] Milanese, U., 2003. Zur Implementierung eines ROBDD-basierten Systems für die Manipulation und Visualisierung von Relationen. Ph.D. thesis, Universität Kiel.

[32] von Neumann, J., Morgenstern, O., 1944. Theory of Games and Economic Behaviour. Princeton University Press.

[33] Peleg, B., 1981. Coalition formation in simple games with dominant players. International Journal of Game Theory 10, 11-33.

[34] Peleg, B., Sudhölter, P., 2003. Introduction to the Theory of Cooperative Games. Springer-Verlag, New York.

[35] Prasad, K., Kelly, J.S., 1990. NP-completeness of some problems concerning voting games. International Journal of Game Theory 19, 1-9.

[36] van Roozendaal, P., 1990. Centre parties and coalition cabinet formations: a game theoretic approach. European Journal of Political Research 18, 325-348.

[37] van Roozendaal, P., 1992a. The effect of dominant and central parties on cabinet composition and durability. Legislative Studies Quarterly 17, 5-36.

[38] van Roozendaal, P., 1992b. Cabinets in Multiparty Democracies. Amsterdam: Thesis Publishers.

[39] van Roozendaal, P., 1993. Cabinets in the Netherlands (1918-1990): The importance of 'dominant' and 'central' parties. European Journal of Political Research 23, 35-54.

[40] van Roozendaal, P., 1997. Government survival in Western multi-party democracies. The effect of credible exit threats via dominance. European Journal of Political Research 32, 71-92.

[41] Schmidt, G., Ströhlein, T., 1993. Relations and Graphs, Discrete Mathematics for Computer Scientists, EATCS Monographs on Theoretical Computer Science, Springer.

[42] Shapley, L.S., 1962. Simple games: an outline of the descriptive theory. Behavioral Science 7, 59-66.

[43] Shapley, L.S., Shubik, M., 1954. A method for evaluating the distribution of power in a committee system. American Political Science Review 48, 787-792.

[44] Szymanski, O., 2003. Relationale Algebra im dreidimensionalen Software-Entwurf - ein werkzeugbasierter Ansatz. M.Sc. thesis, Universität Dortmund.

[45] Vailant J.G., 1979. The complexity of computing the permanent. Theoretical Computer Science 8, $189-201$. 